
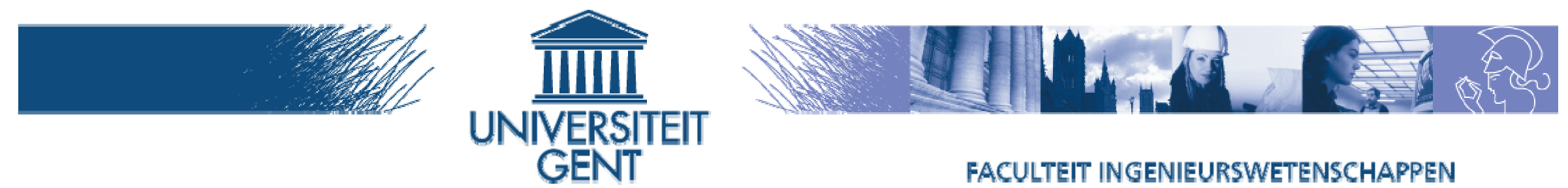

FACULTEIT INGENIEURSWETENSCHAPPEN

\title{
Indoor off-body communication based on a textile multi-antenna system integrated in clothing for rescue workers
}

Hendrik Rogier, Carla Hertleer, Luigi Vallozzi, Patrick Van Torre, Frederick Declercq and Marc Moeneclaey

Information Technology Department, Ghent University, St.-Pietersnieuwstraat 41, B-9000 Ghent, Belgium, Hendrik.Rogier@intec.UGent.be 


\section{Outline}

- Introduction

- Advanced e-textiles for firefighters and civilian victims (ProeTex) project

- Textile multi-antenna system

- Circular-polarized textile antenna

- Design

- Performance

- Return loss, Gain (anechoic chamber)

- Off-body wireless link (antenna in suit of fully-equipped rescue worker)

- Dual-polarized antenna textile antenna

- Design

- Performance

- Conclusions 


\section{Introduction: Proetex project}

\section{- Firemen have excellent protective clothing}

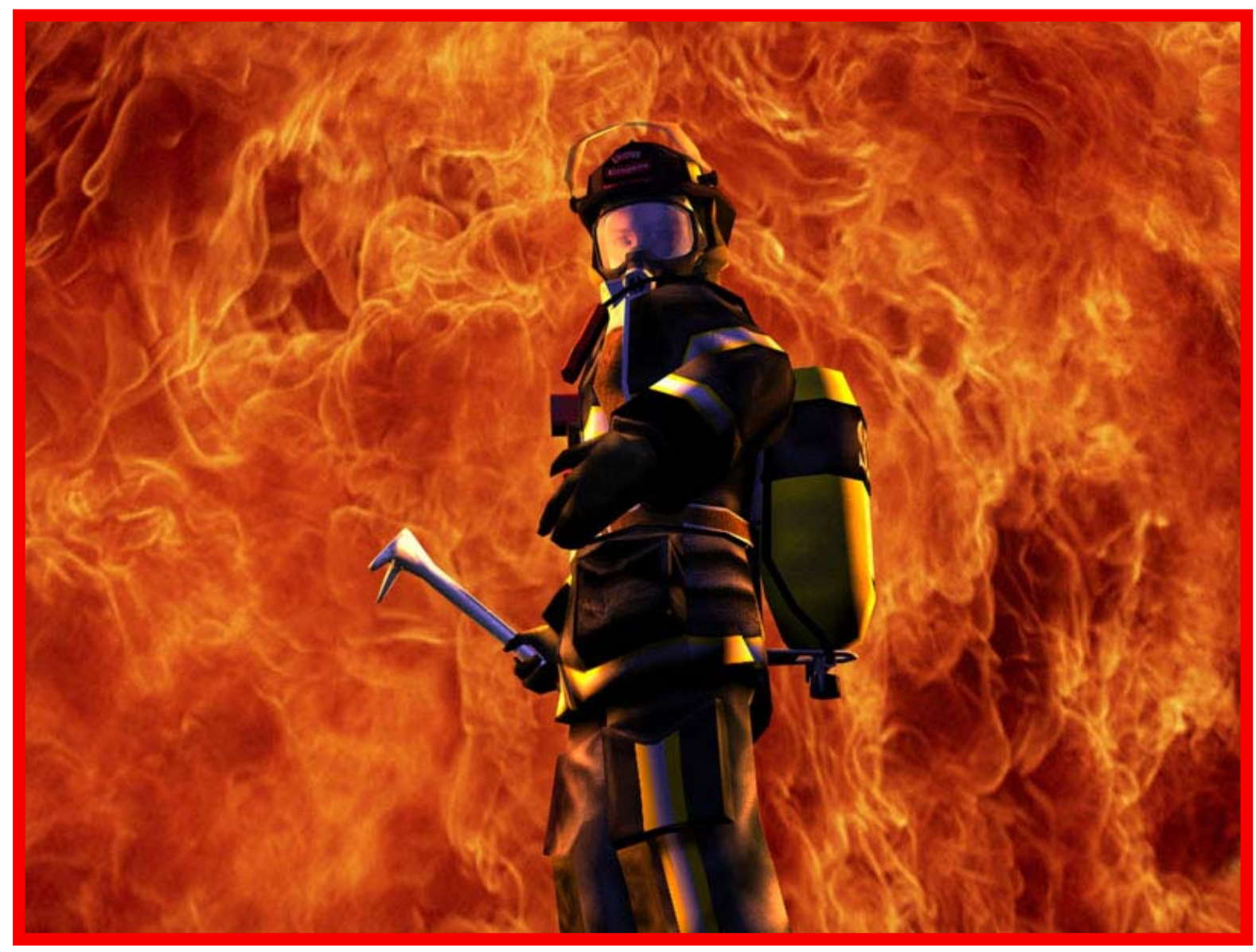




\title{
Introduction: Proetex project
}

\section{- Yet, protection and safety can be improved}

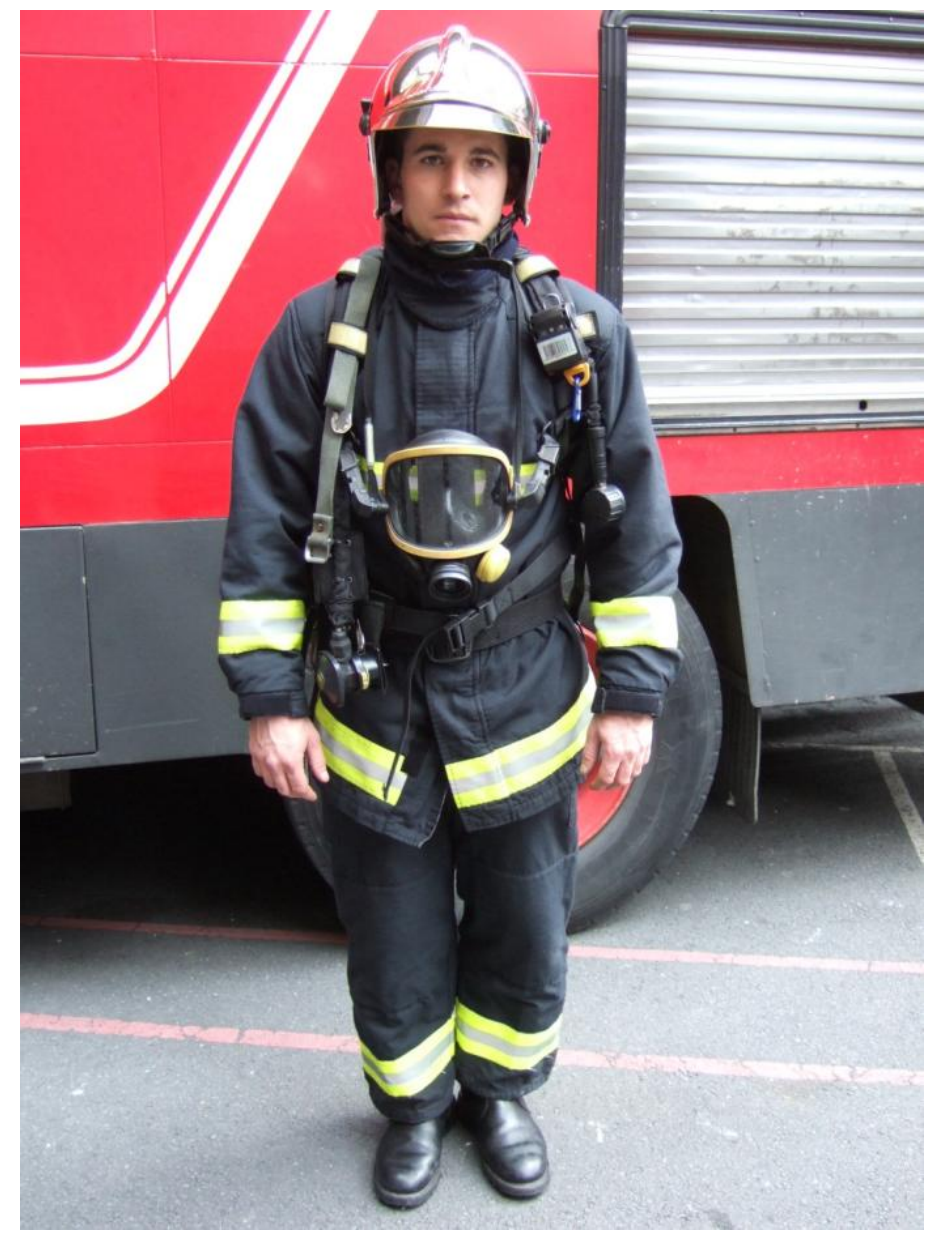

\author{
Textiles + Electronics \\ $=$
}

\section{Wearable Textile System}

For

Sensing

Interconnecting

Communicating

Powering

Actuating 
Intheduction: Proetex project

\section{- Wearable textile system consists of}

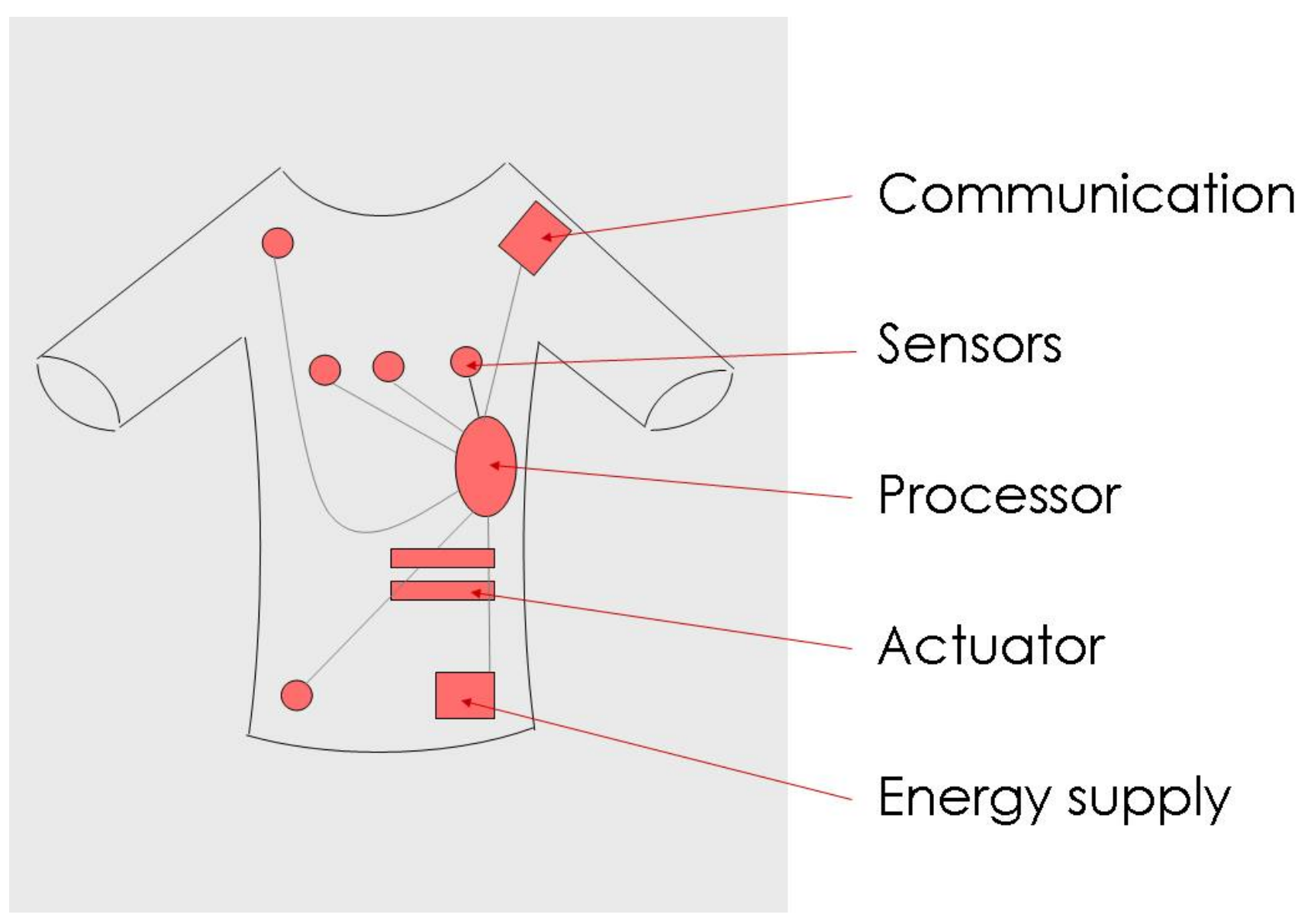




\section{Introduction: Proetex project}

\section{- ProeTex wearable textile system}

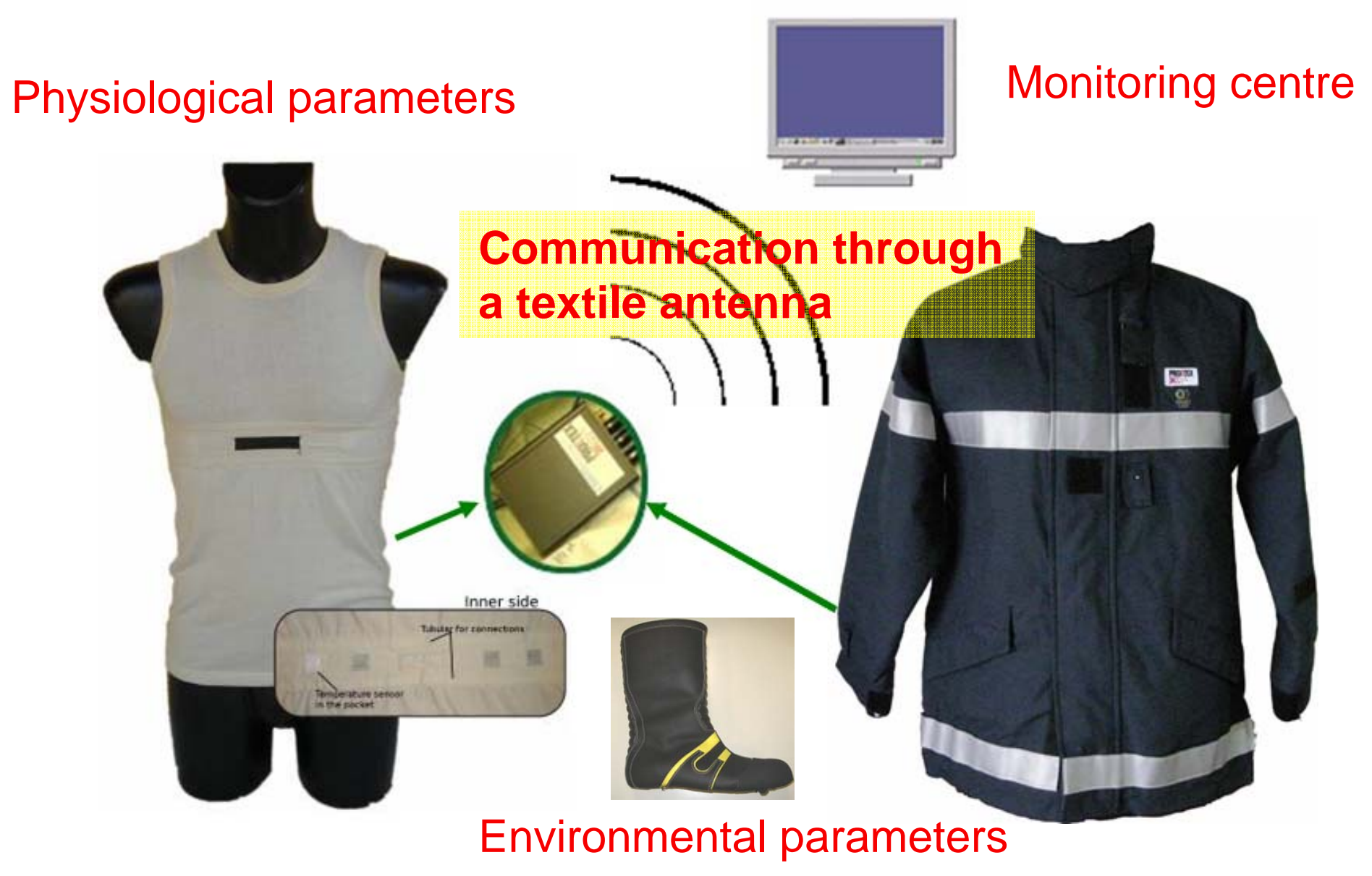




\section{Introduction: Proetex project}

\section{- First inner and outer garment prototypes}

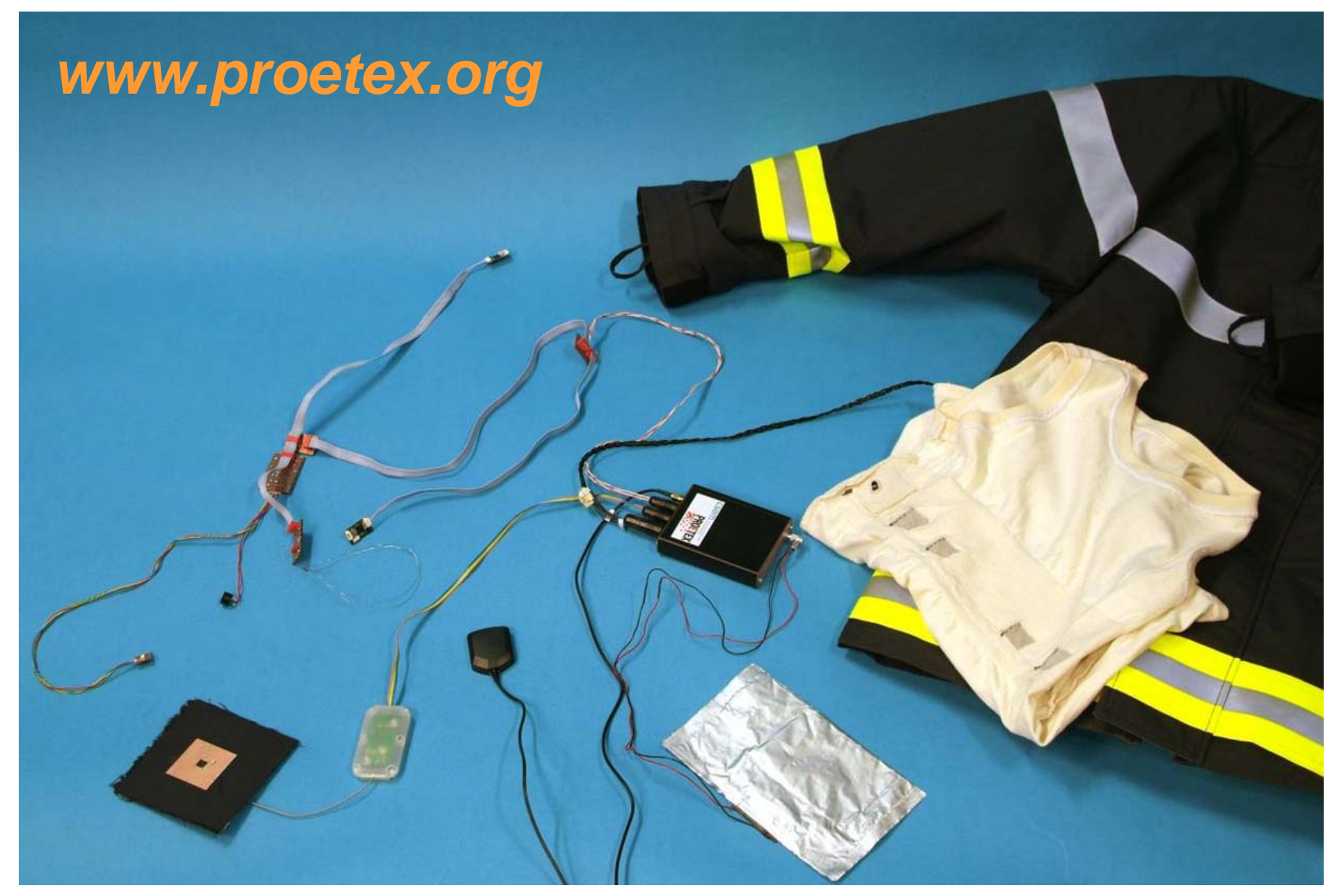




\section{Textile antenna: Design}

\section{- Microstrip patch antenna}

- Low profile + easy to integrate

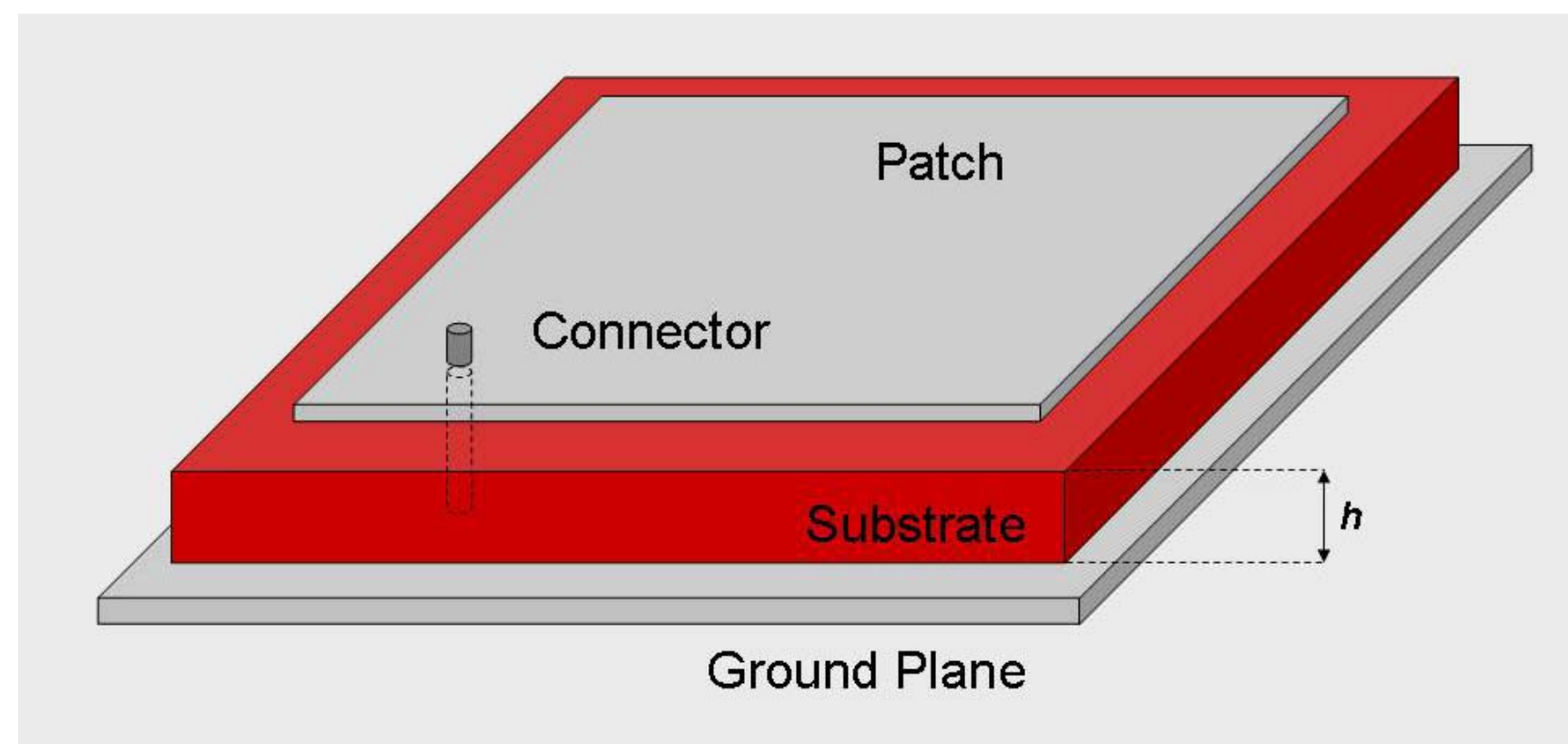

\section{- Textile materials:}

- Breathable + comfortable to wear 


\section{Textile antenna: Design}

\section{- Communication in $2.45 \mathrm{GHz}$ ISM band}

- Use of existing protocols, e.g. WiFi

- Use more transmit power during emergencies (>20dBm) to cover larger distances

\section{- Design criterion}

- antenna operating in $2.4-2.4835 \mathrm{GHz}$ range

- -10dB return loss in entire ISM-band (90\% power injected)

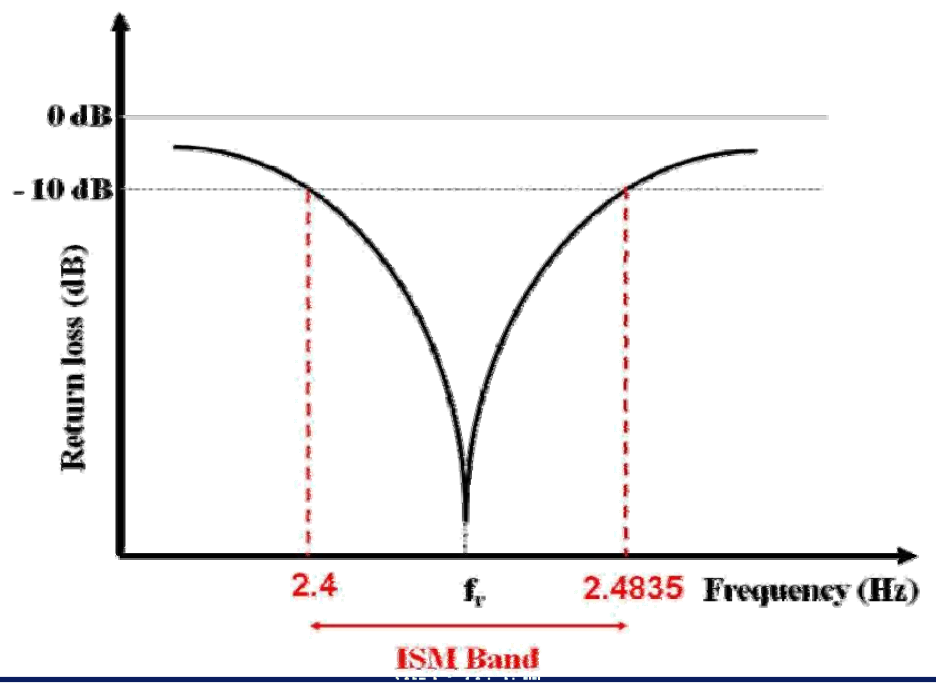




\section{Textile antenna: Design}

\section{- Antenna substrate: protective foam}

- Commonly used in fire-fighter garments

- flexible, shock-absorbing, fire-retarding, moisture repellent

- Thickness: 5.5mm

- uniform thickness, even after compression

- large bandwidth

- moisture regain (MR) of $0.84 \%$

$$
\text { moisture regain }=\frac{\text { conditioned weight }- \text { dry weight }}{\text { dry weight }} 100 \%
$$

- stable antenna characteristics 


\section{Textile antenna: Design}

\section{- Antenna topology: rectangular ring}

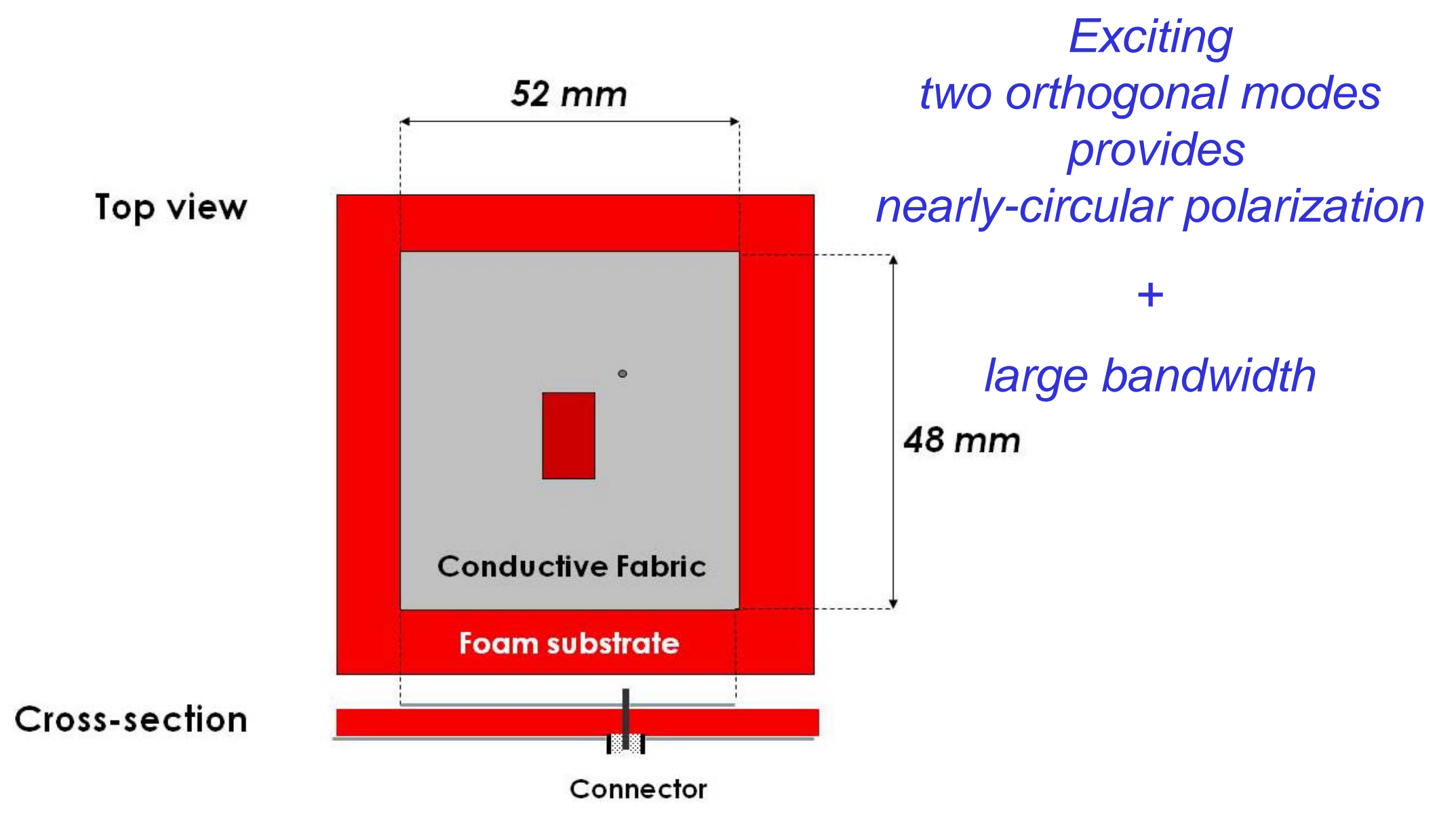




\section{Textile antenna prototype}
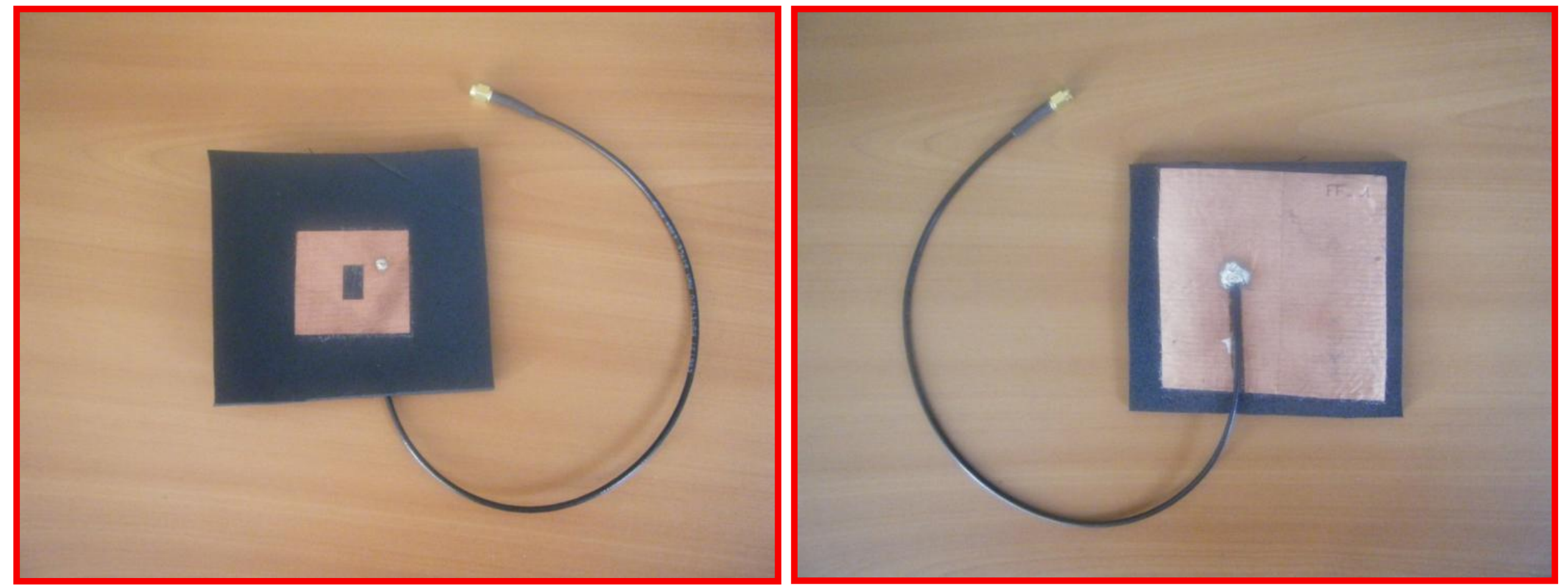

The antenna patch, radiating away from the person
The ground plane, improving radiation away from the person and shielding the body from radiation

\section{A flexible cable,}

to connect to the electronic box 


\section{Textile antenna performance}

- Reflection measurements

- check impedance bandwidth

- Moisture influence

- evaluate effect relative humidity in jacket

- Transmission measurements

- anechoic chamber:

- antenna gain

- antenna efficiency

- Real-life scenario - antenna in fireman suit

- indoor environment:

- Signal-to-Noise Ratio

- Bit-Error-Rate 


\section{Textile antenna performance}

\section{- Reflection measurements}

- Impedance bandwidth: ISM band covered

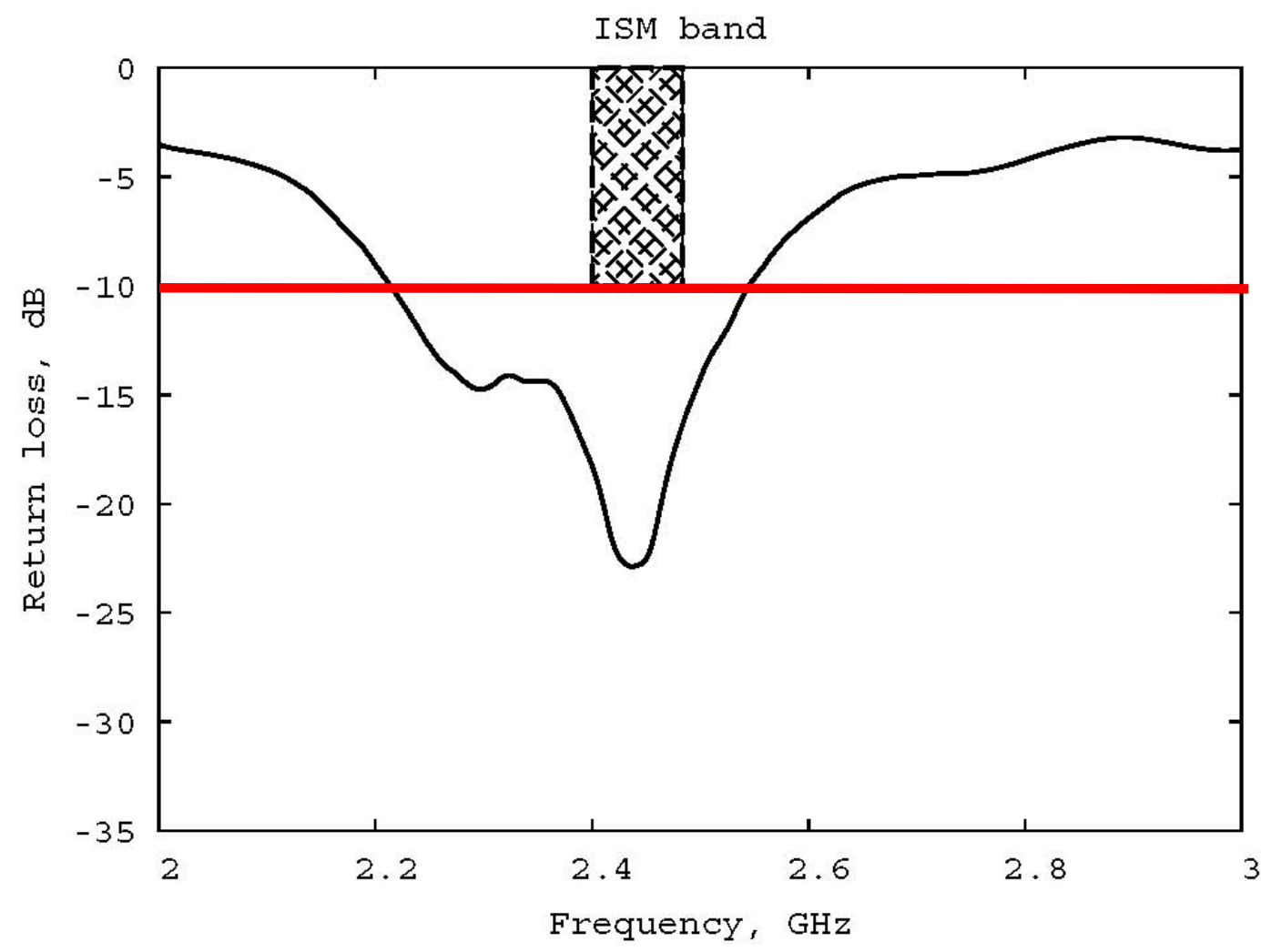




\section{Textile antenna performance}

\section{- Effect of humidity}

- Changing humidity alters reflection characteristic only slightly (Foam MR =0,84\%)

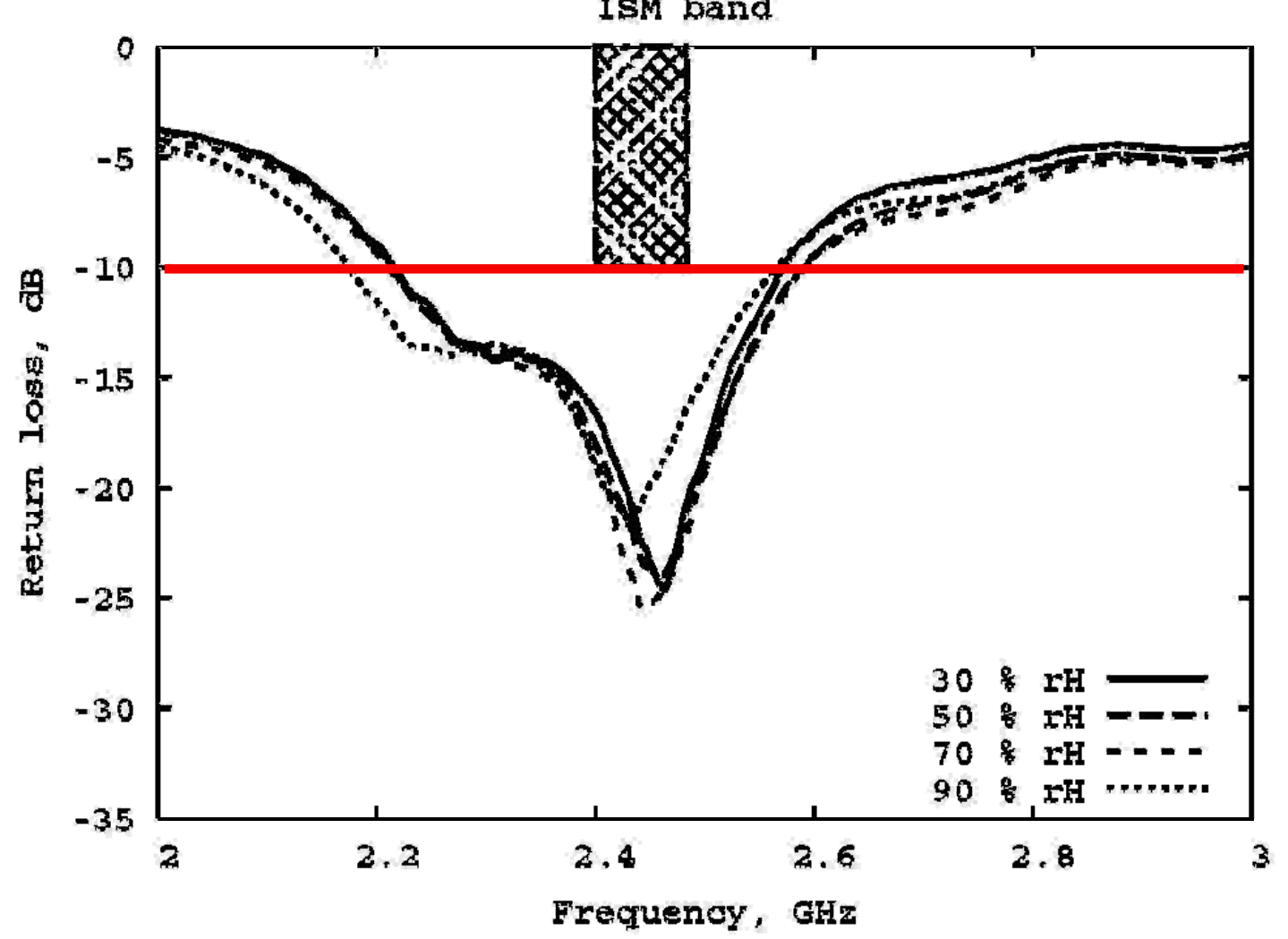




\section{Textile antenna performance}

\section{- Effect of humidity}

- For comparison:

cotton as substrate material $(\mathrm{MR}=7.33 \%)$

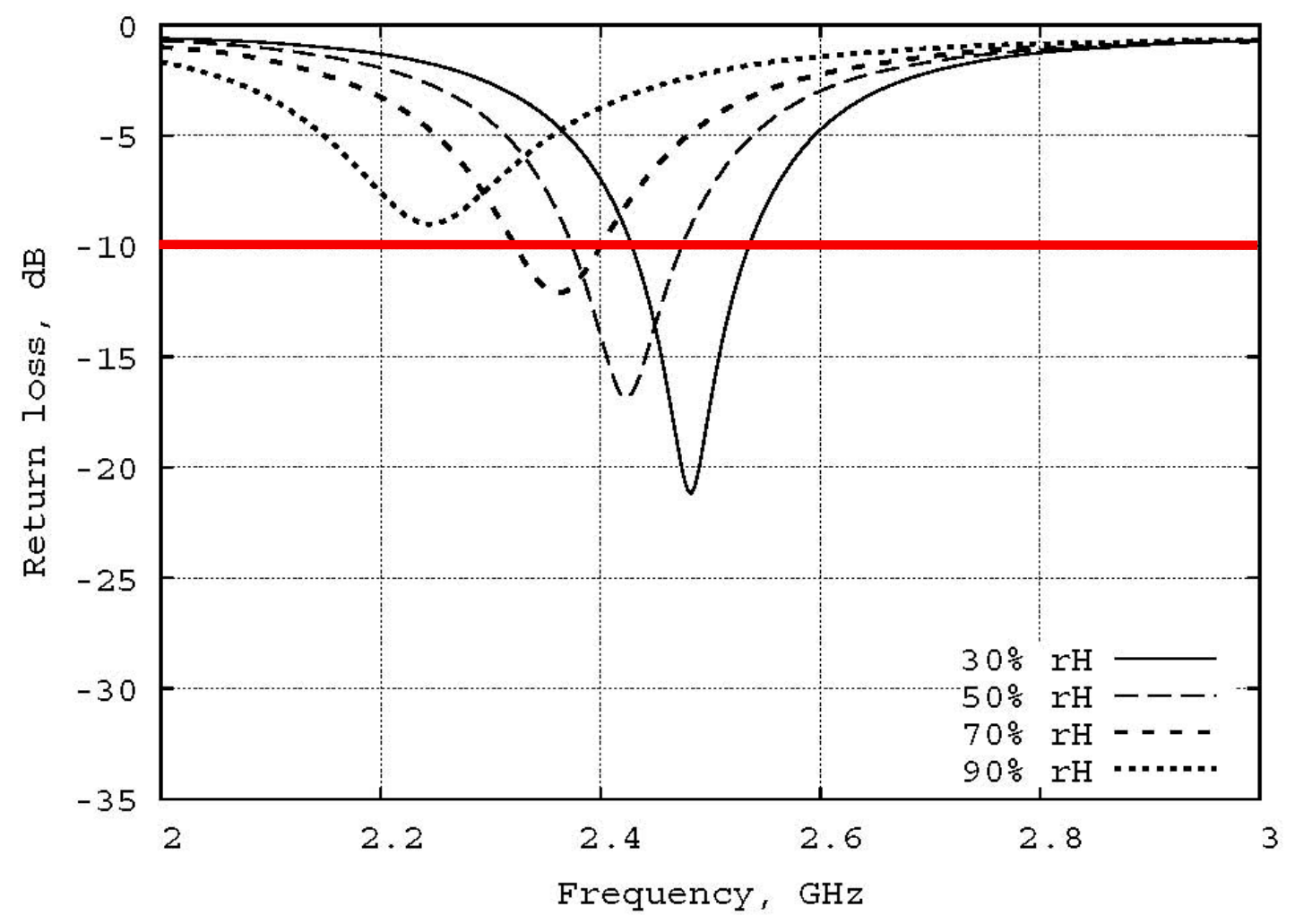




\section{Textile antenna performance}

\section{- Transmission measurements}

\section{- anechoic chamber}

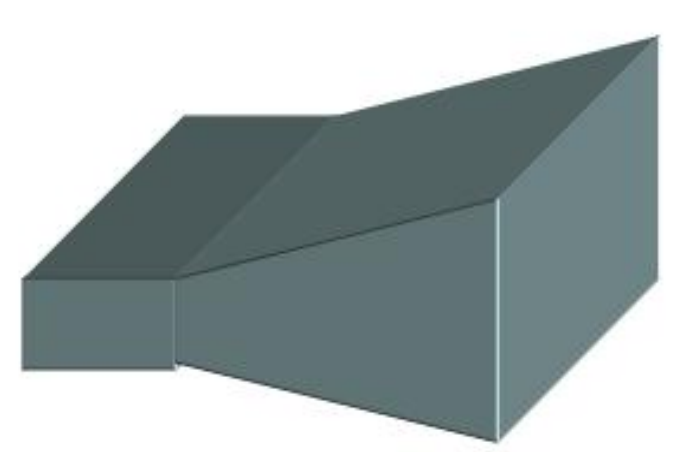

$\mathbf{R}$

Transmitting standard gain horn antenna

Receiving textile antenna 


\section{Textile antenna performance}

\section{- Transmission measurements}

- Antenna gain of $6.7 \mathrm{dBi}$ along broadside

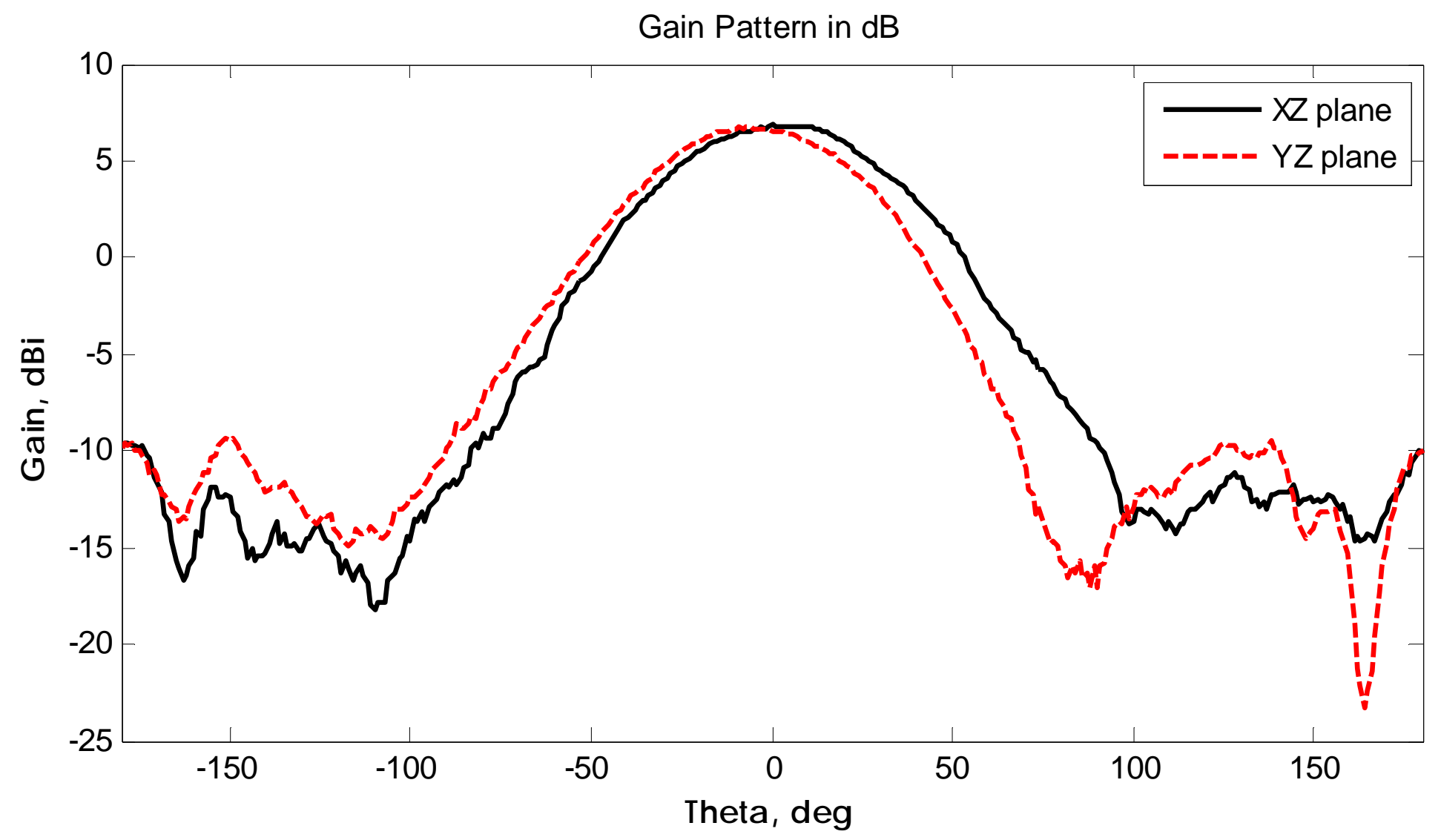




\section{Textile antenna performance}

\section{- Real-life scenario}

- two antennas integrated into fire fighter jacket

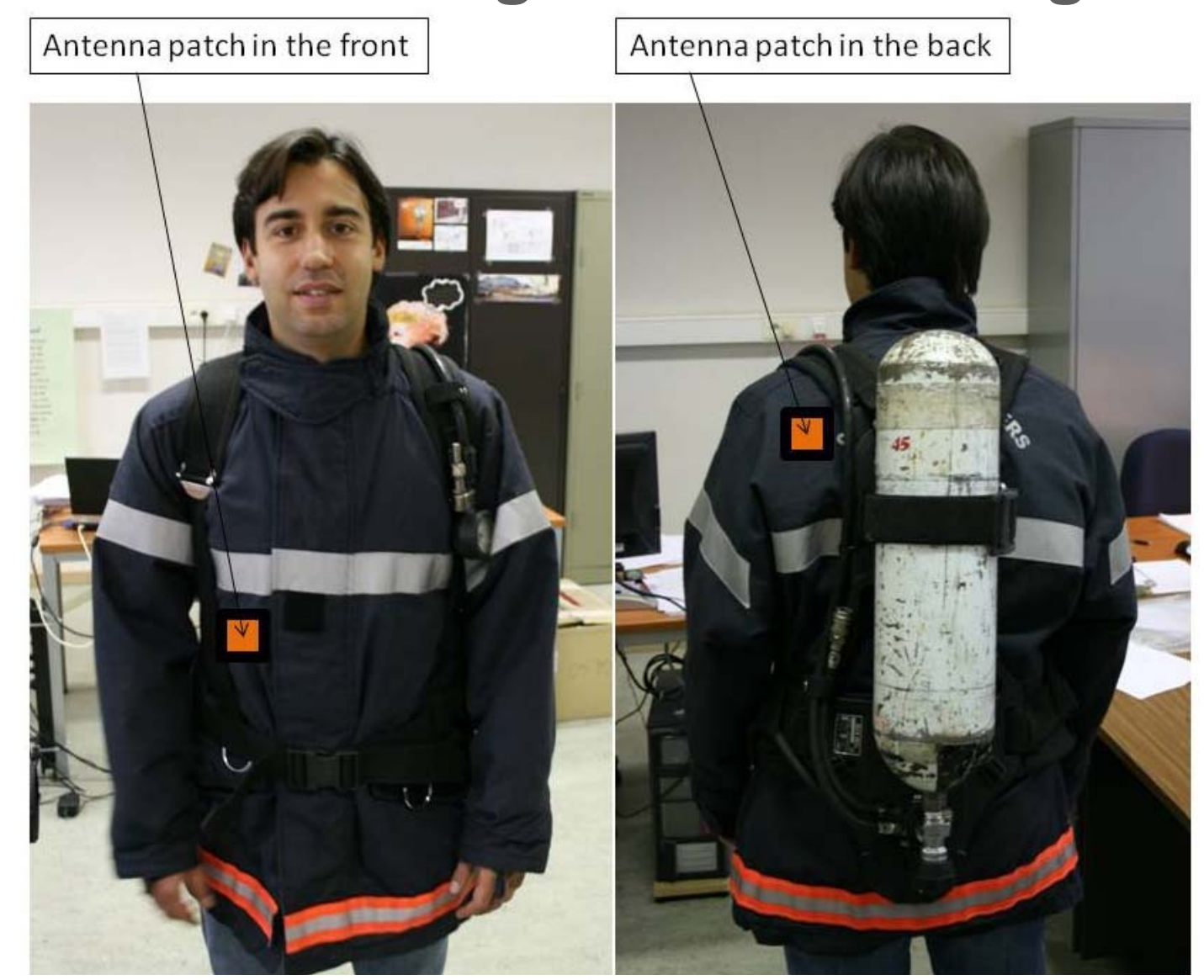




\section{Textile antenna performance}

\section{- Real-life scenario}

- Antenna location: under 2 outermost layers

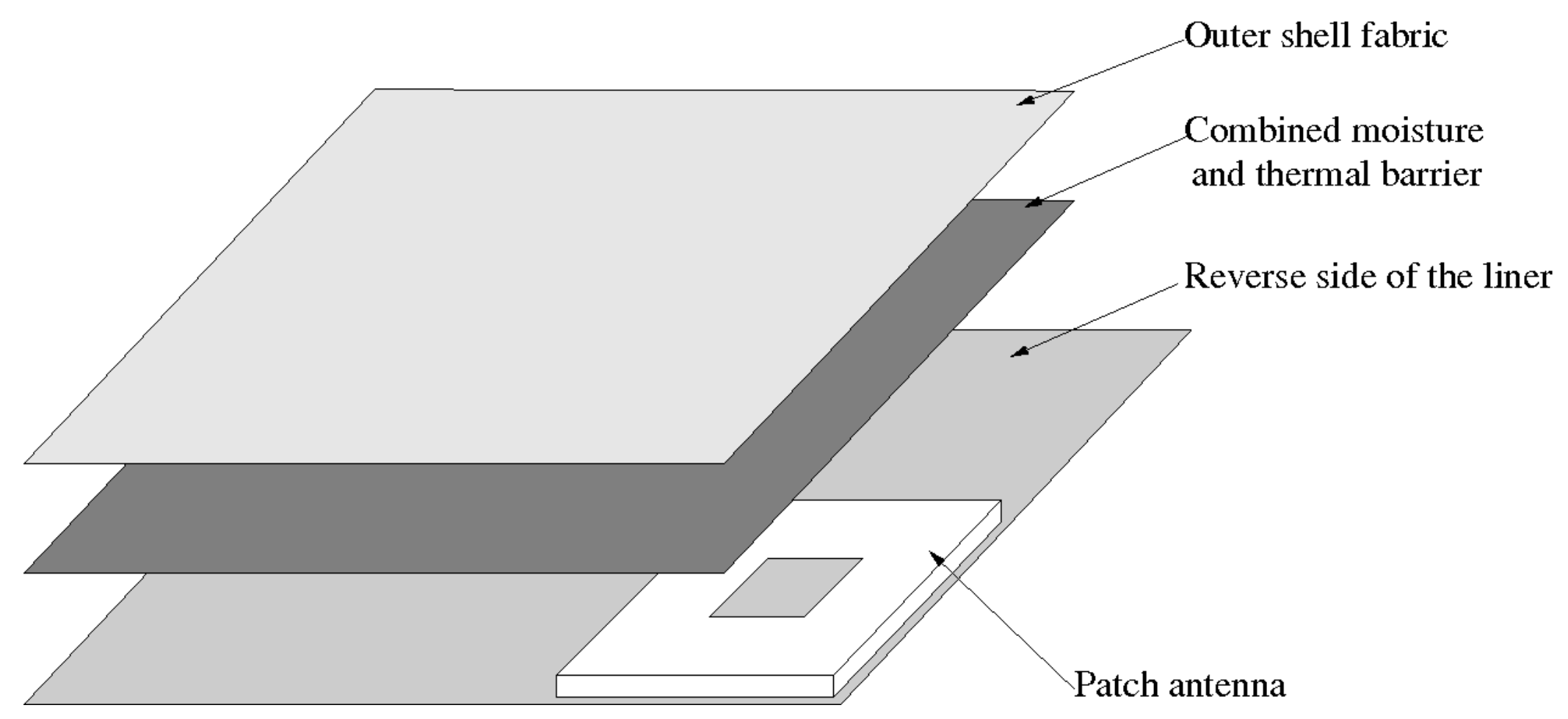

Antenna protected against heat and moisture 


\section{Textile antenna performance}

\section{- Real-life scenario}

- Textile antenna inside the jacket

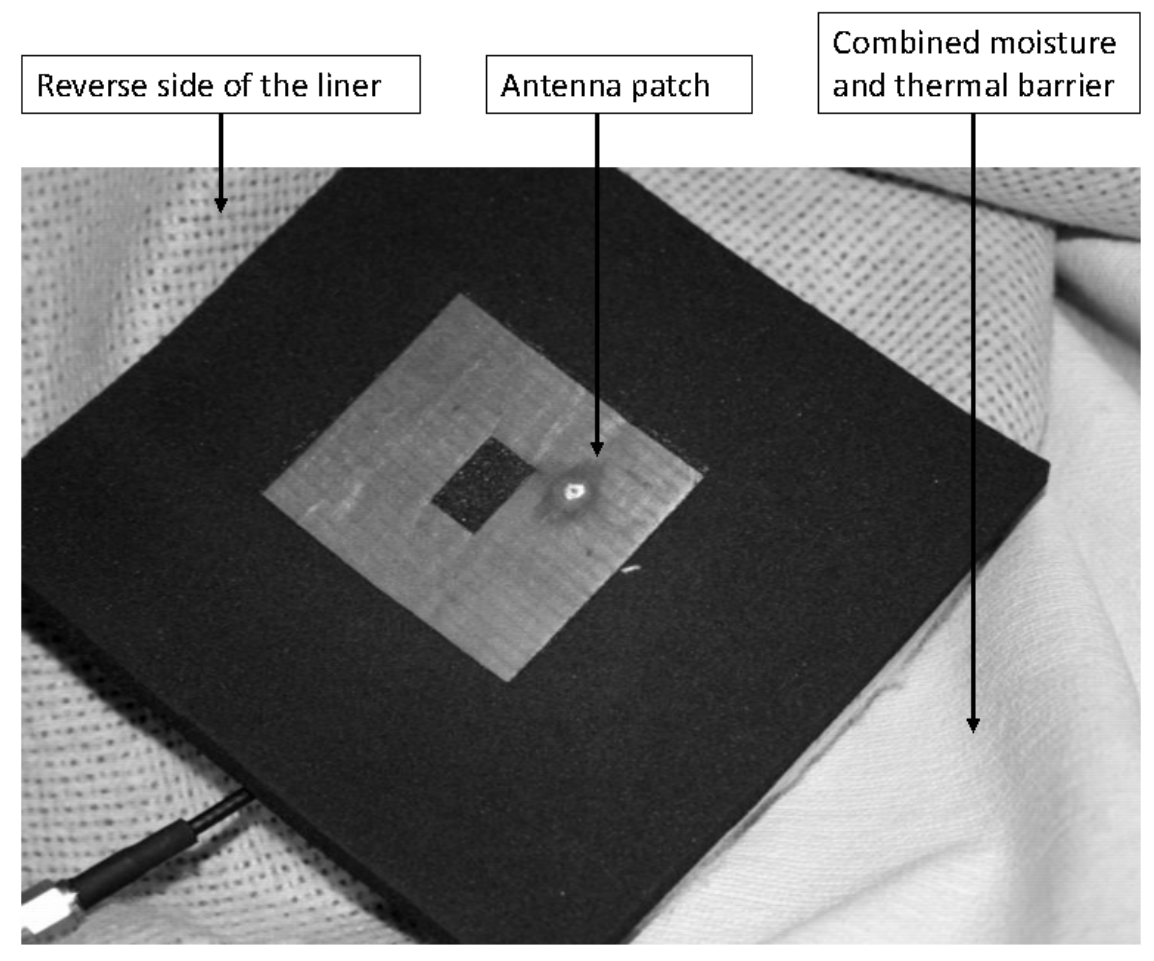

Antenna protected against heat and moisture 


\section{Textile antenna performance}

- Radiation pattern: Effect of the air bottle

- Textile antenna inside the jacket (front side)

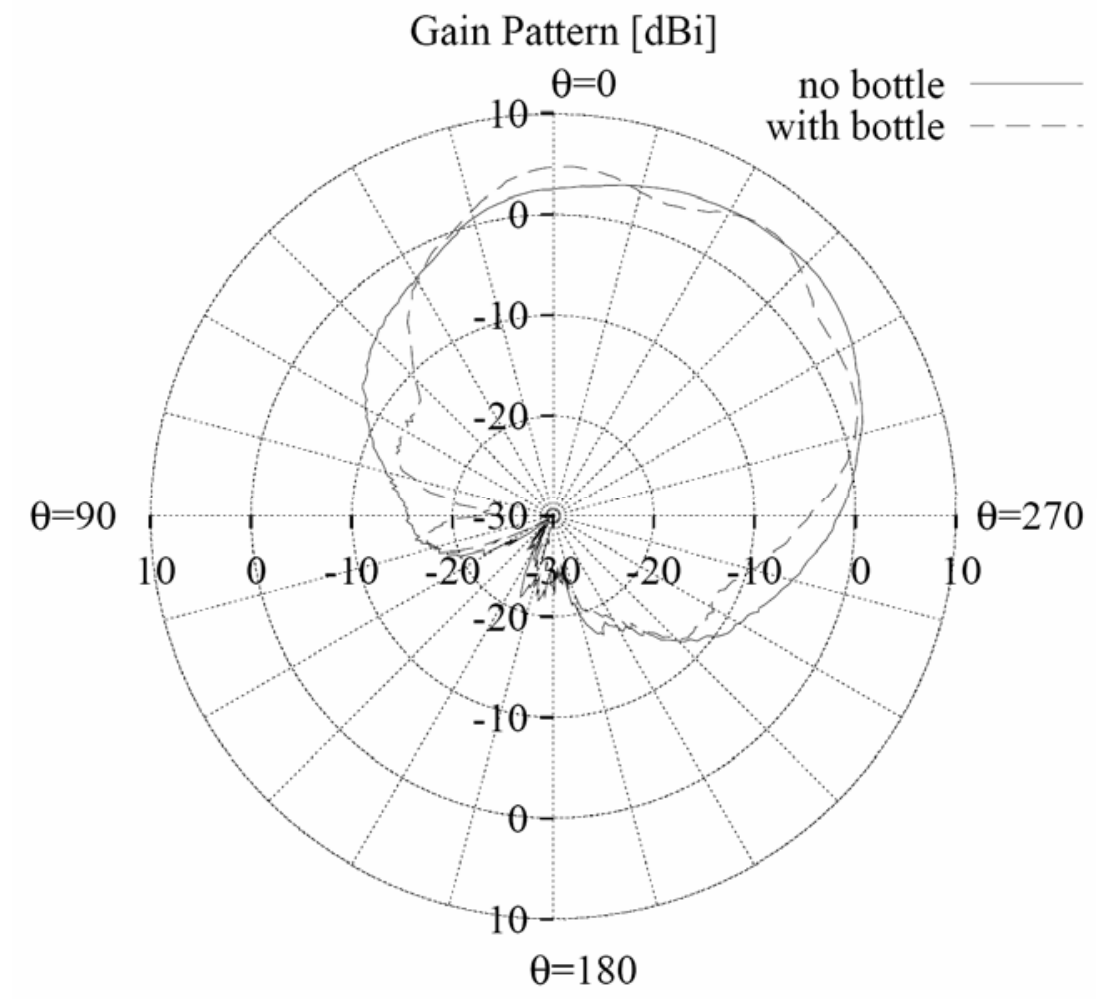

Integration in jacket and presence air bottle do not substantially reduce antenna gain

Direction of maximum gain tilted to $40^{\circ}$ 


\section{Textile antenna performance}

\section{- Real-life scenario}

- Equipped fire fighter in indoor environment
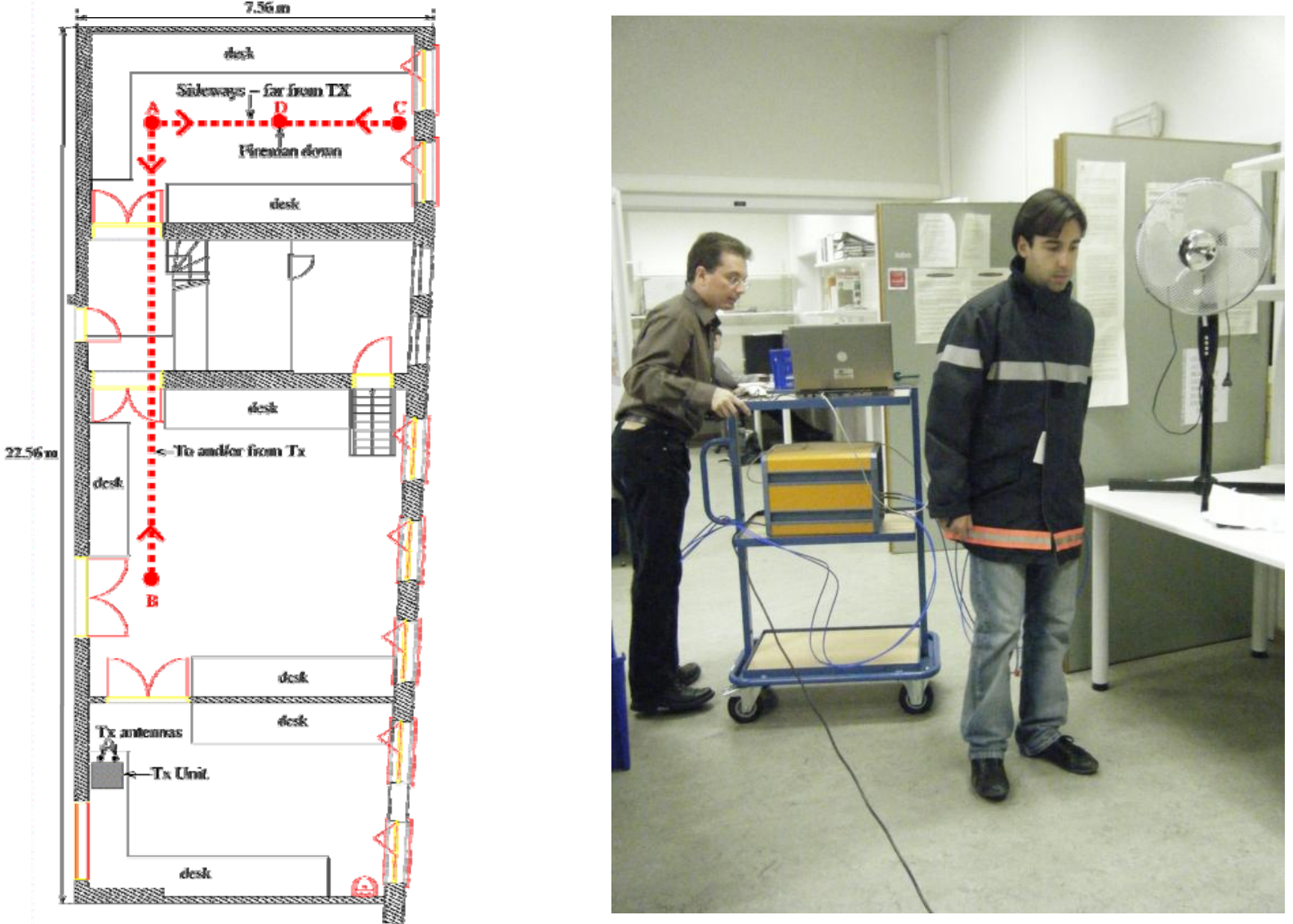


\section{Textile antenna performance}

\section{- Real-life scenario}

- Wireless link: shadowing and fading

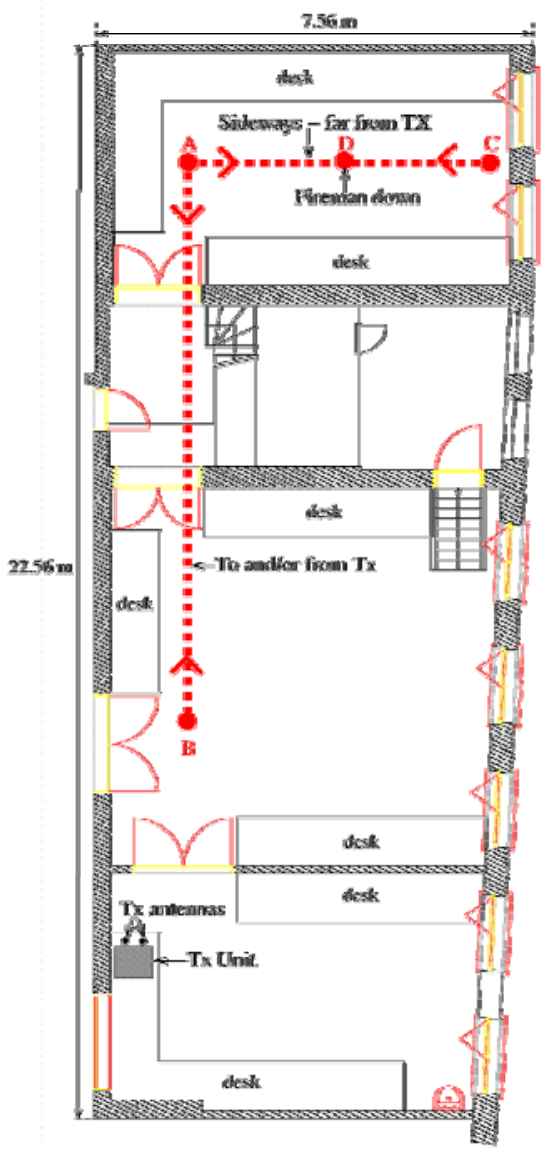

1. fading: multipath radio propagation

2. shadowing by body: hemispherical coverage by single on-body antenna

3. two textile patch antennas, in front and back: full coverage improves communication reliability 


\section{Textile antenna performance}

\section{- Real-life scenario: measured SNR and BER}

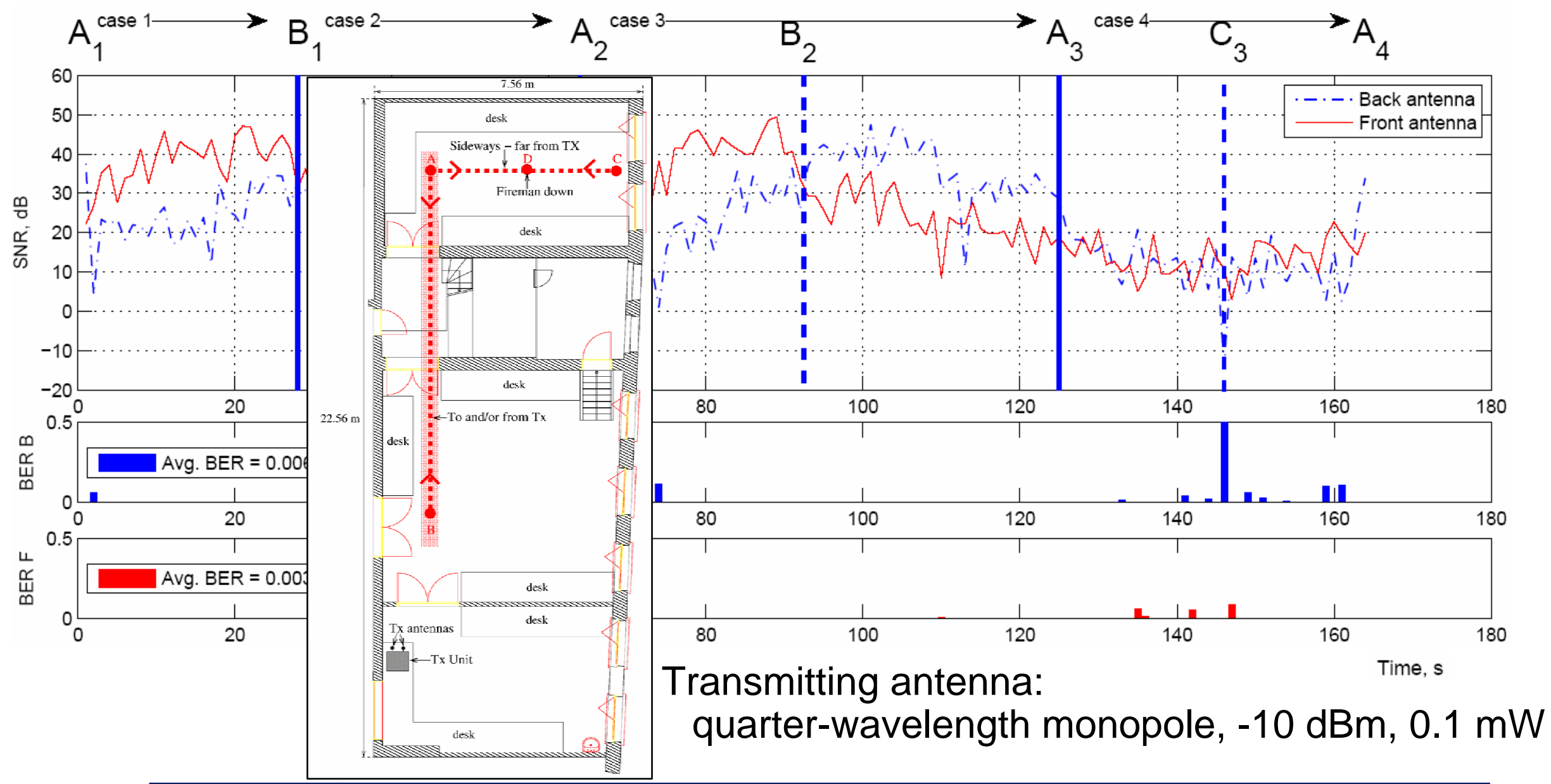




\section{Textile antenna performance}

\section{- Real-life scenario: measured SNR and BER}

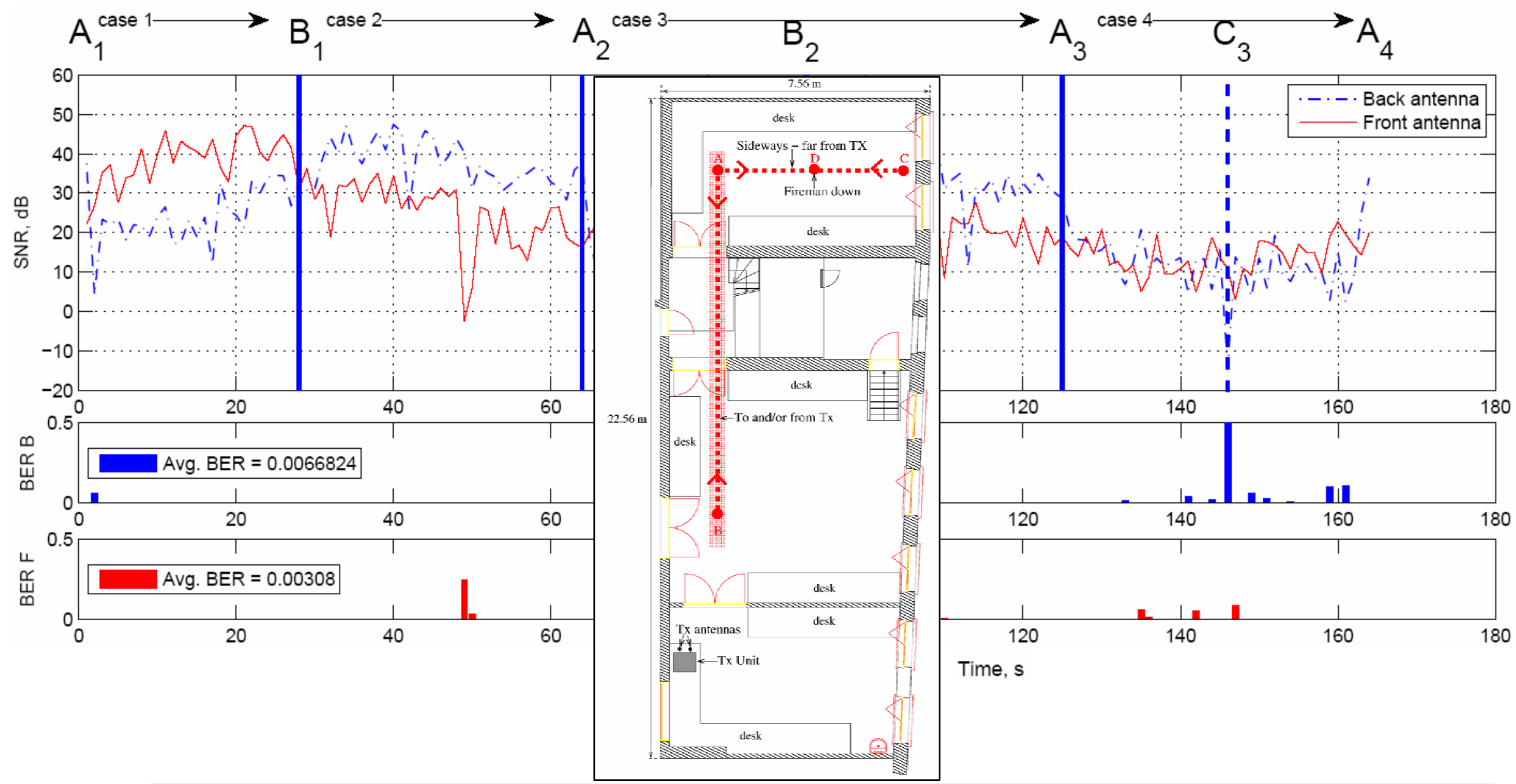




\section{Textile antenna performance}

\section{- Real-life scenario: measured SNR and BER}

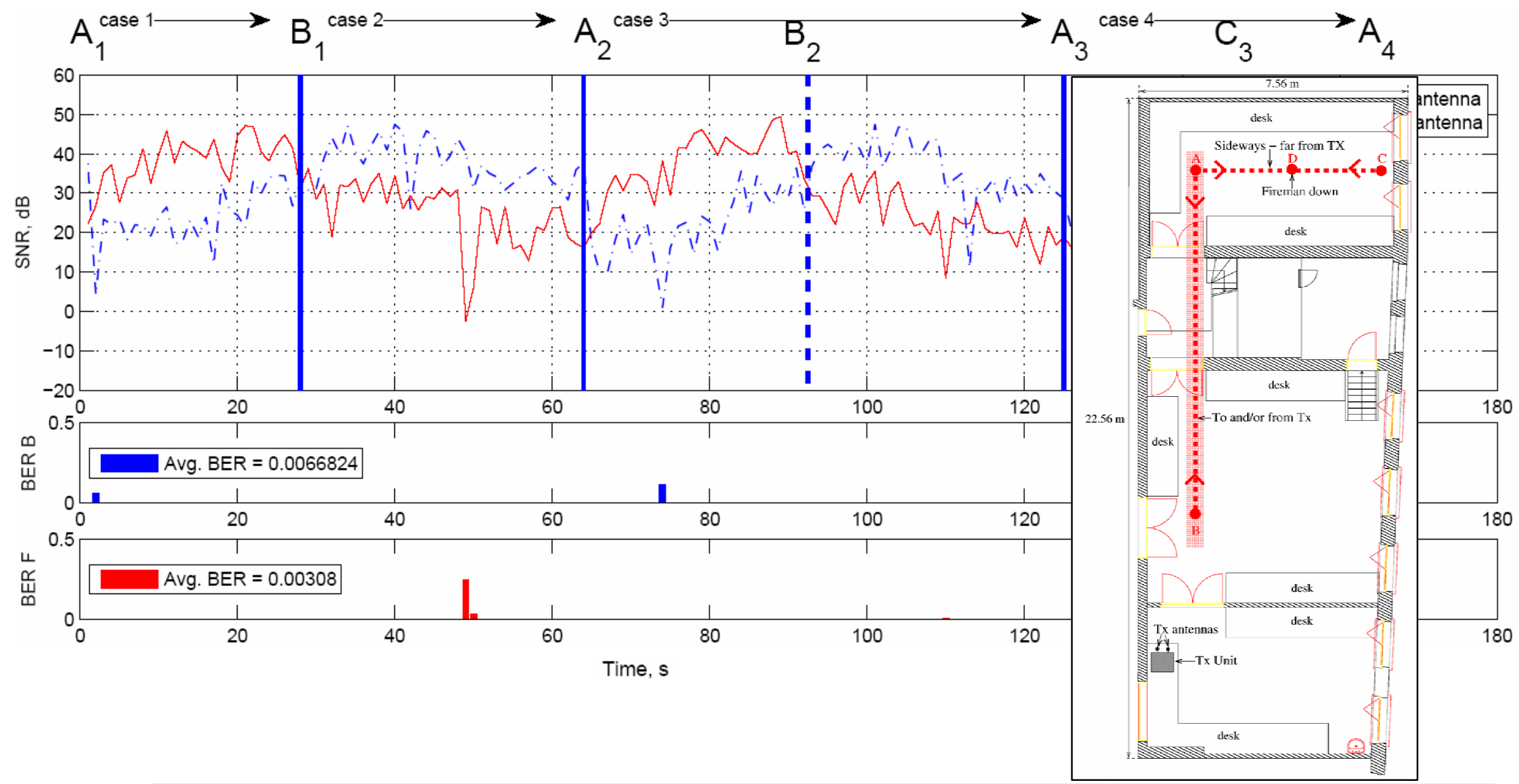




\section{Textile antenna performance}

\section{- Real-life scenario: measured SNR and BER}

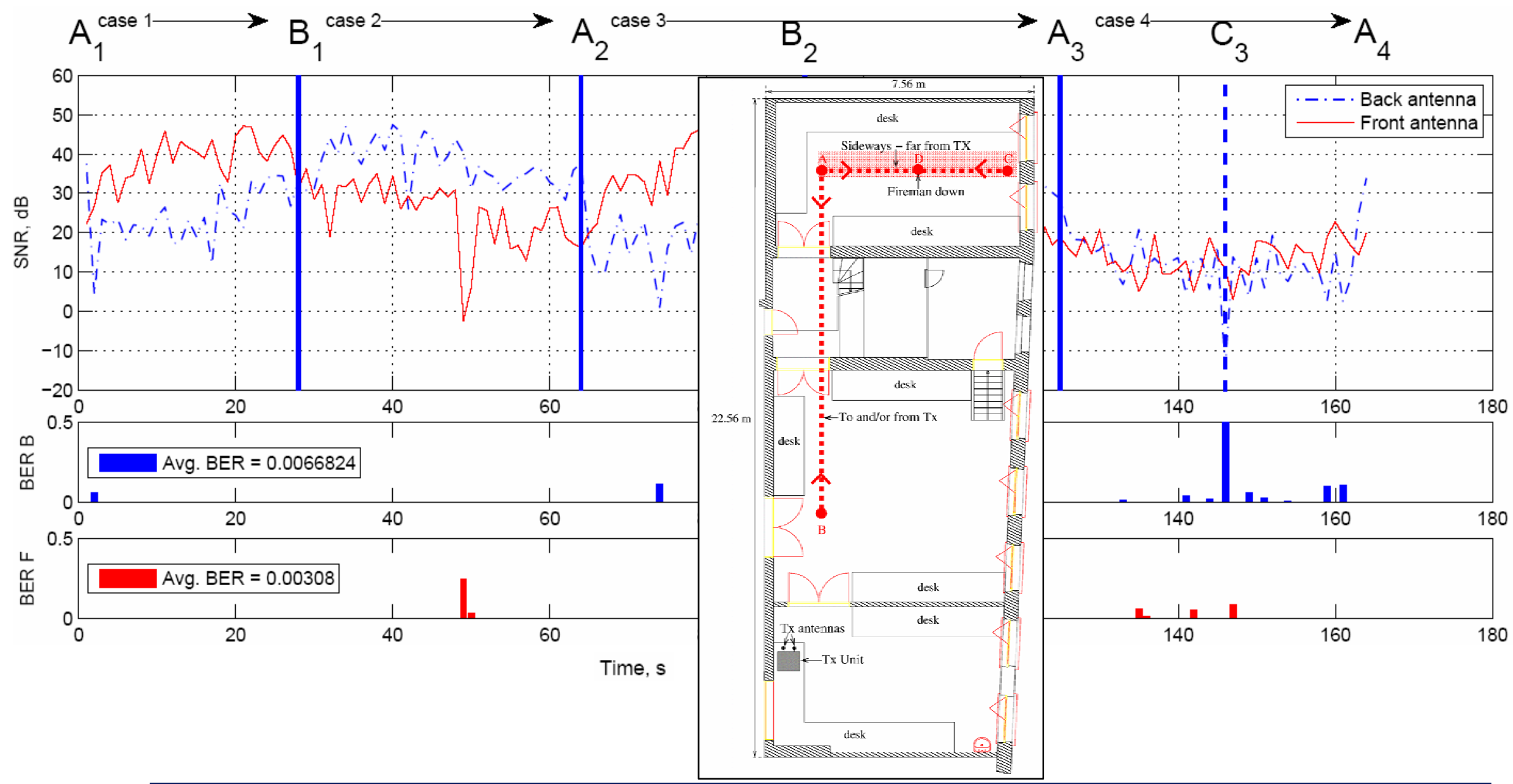




\section{Textile antenna performance}

\section{- Mitigating fading by Selection Combining}

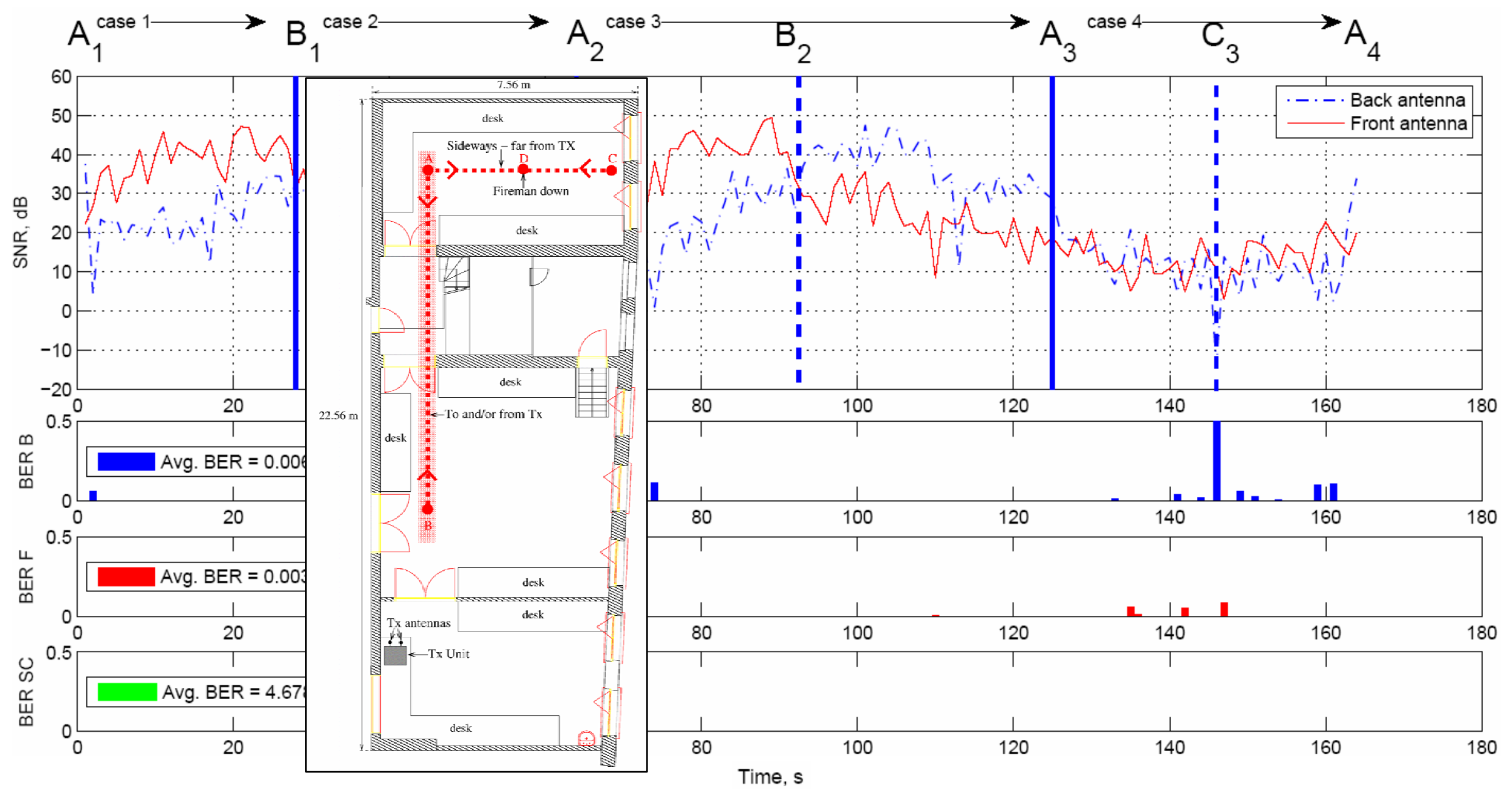




\section{Textile antenna performance}

\section{- Mitigating fading by Selection Combining}

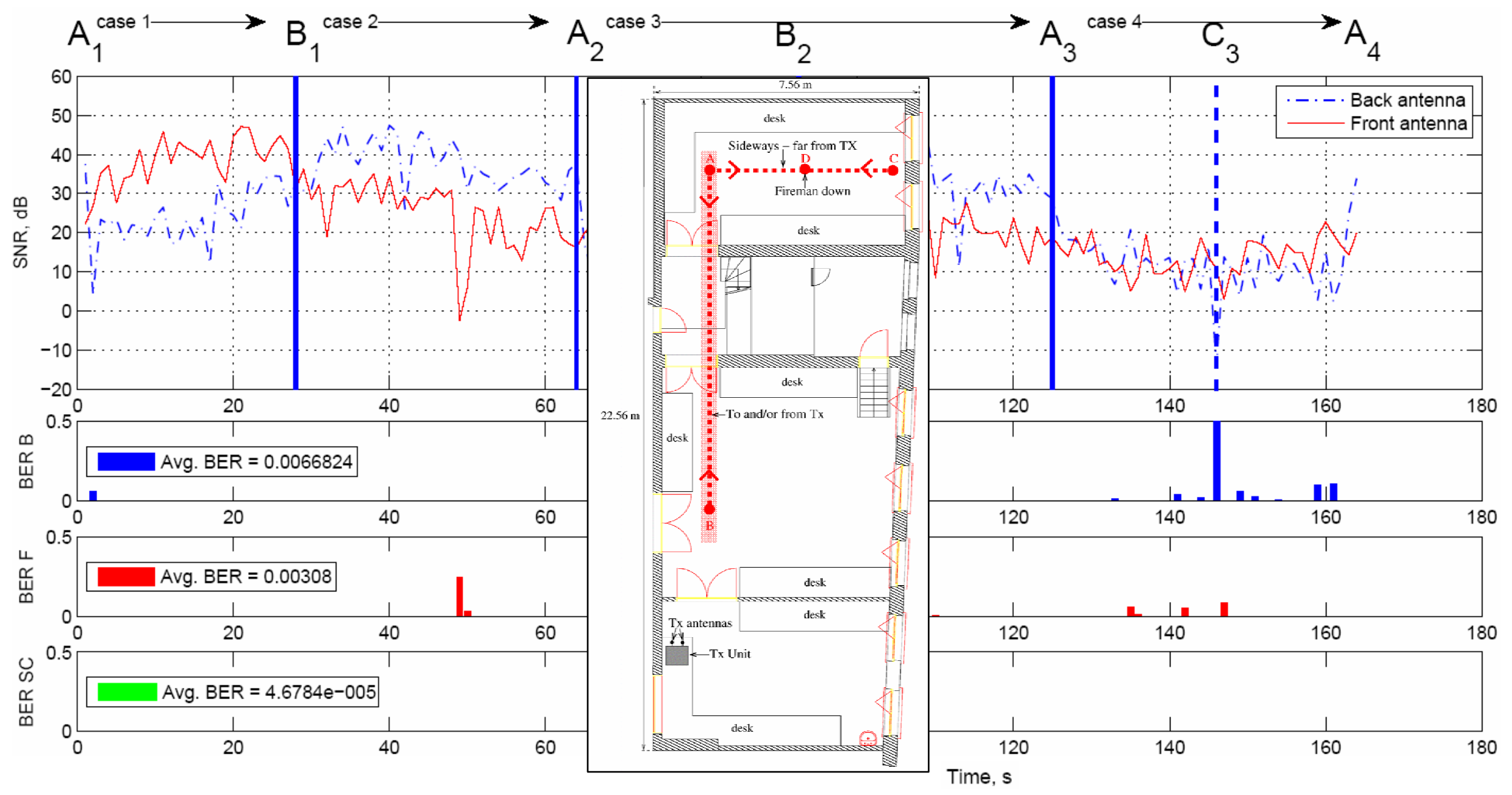




\section{Textile antenna performance}

\section{- Mitigating fading by Selection Combining}

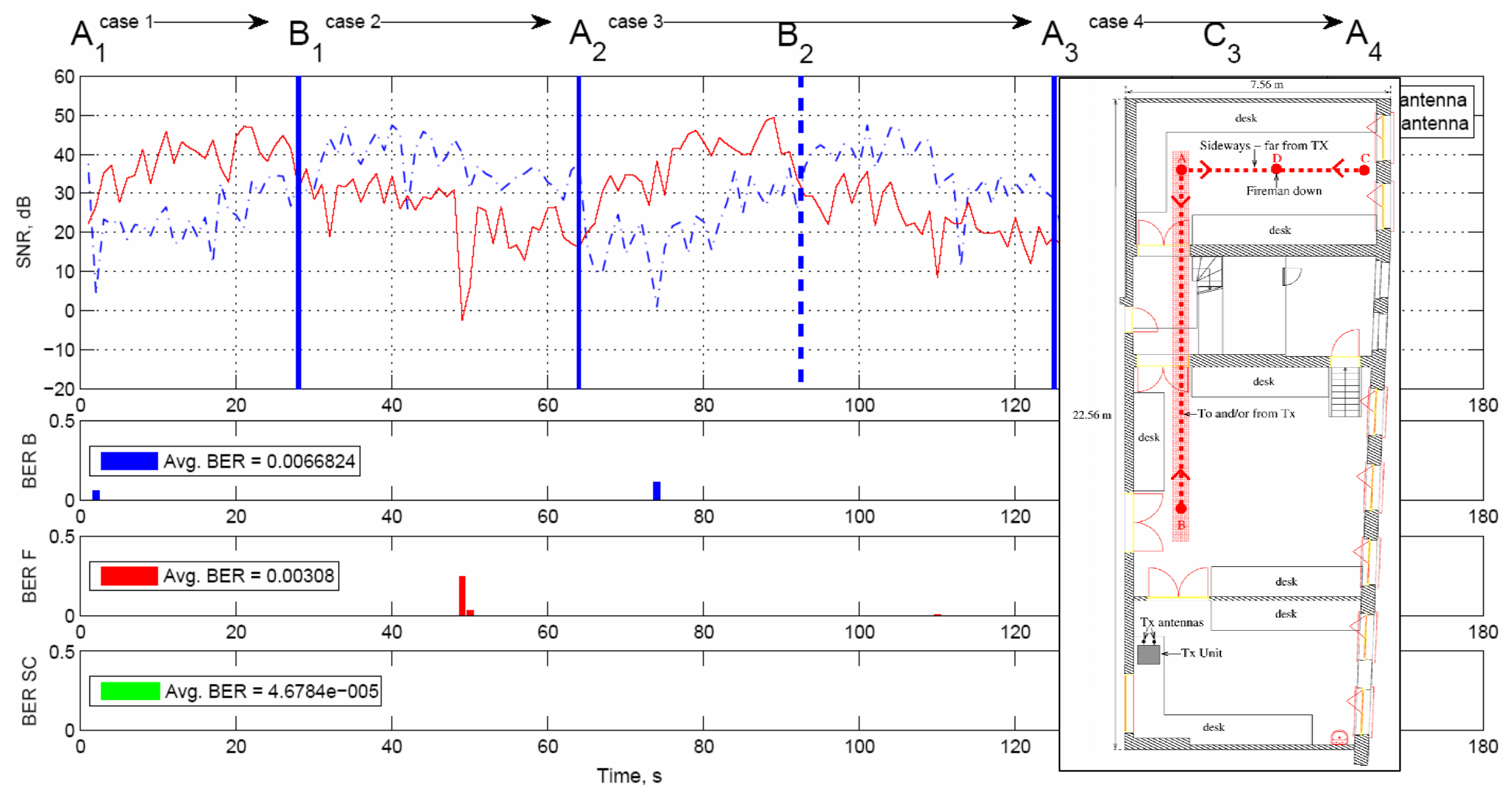




\section{Textile antenna performance}

\section{- Mitigating fading by Selection Combining}

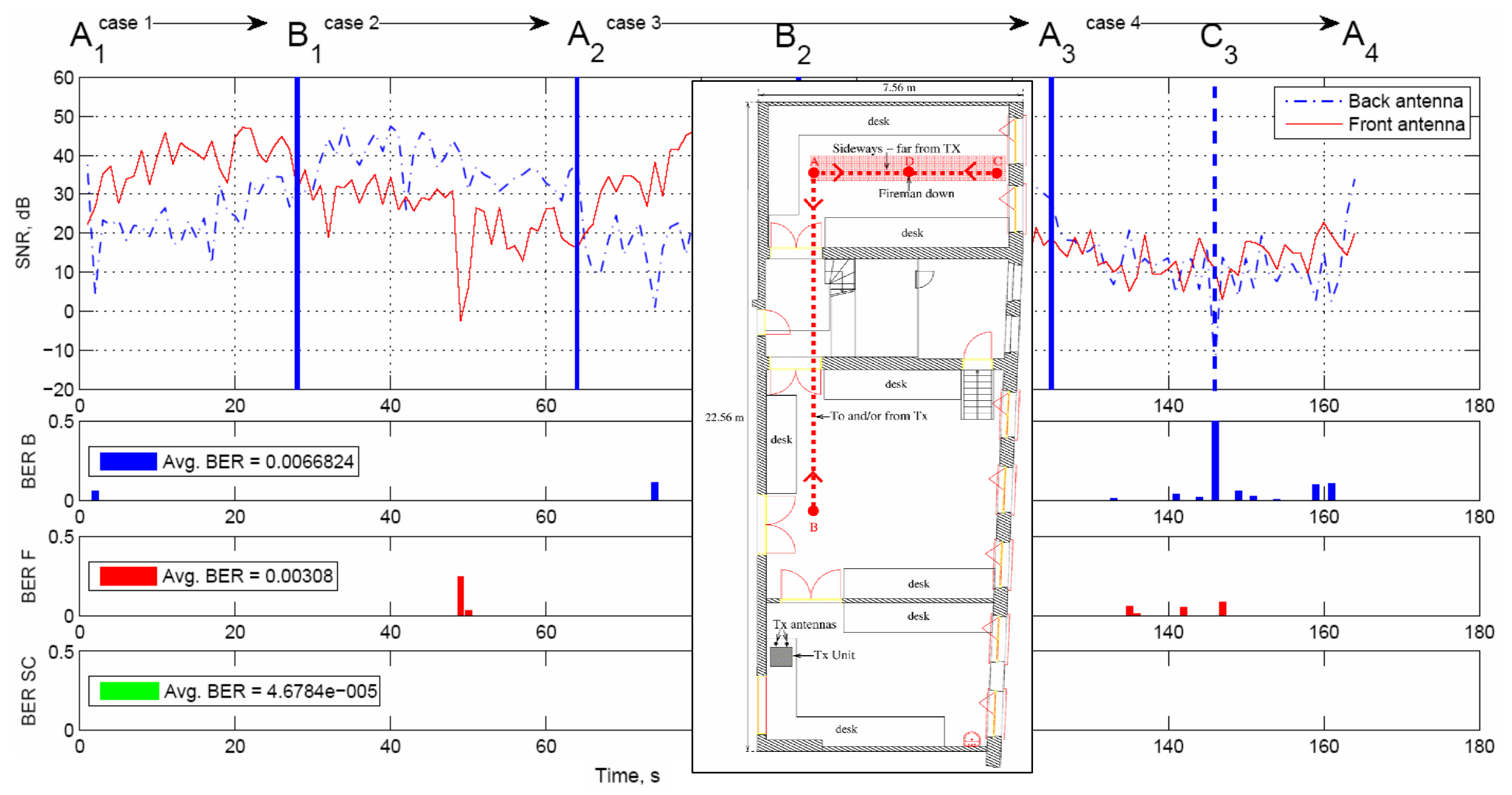




\section{- Mitigating fading by Maximum-Ratio Combining}
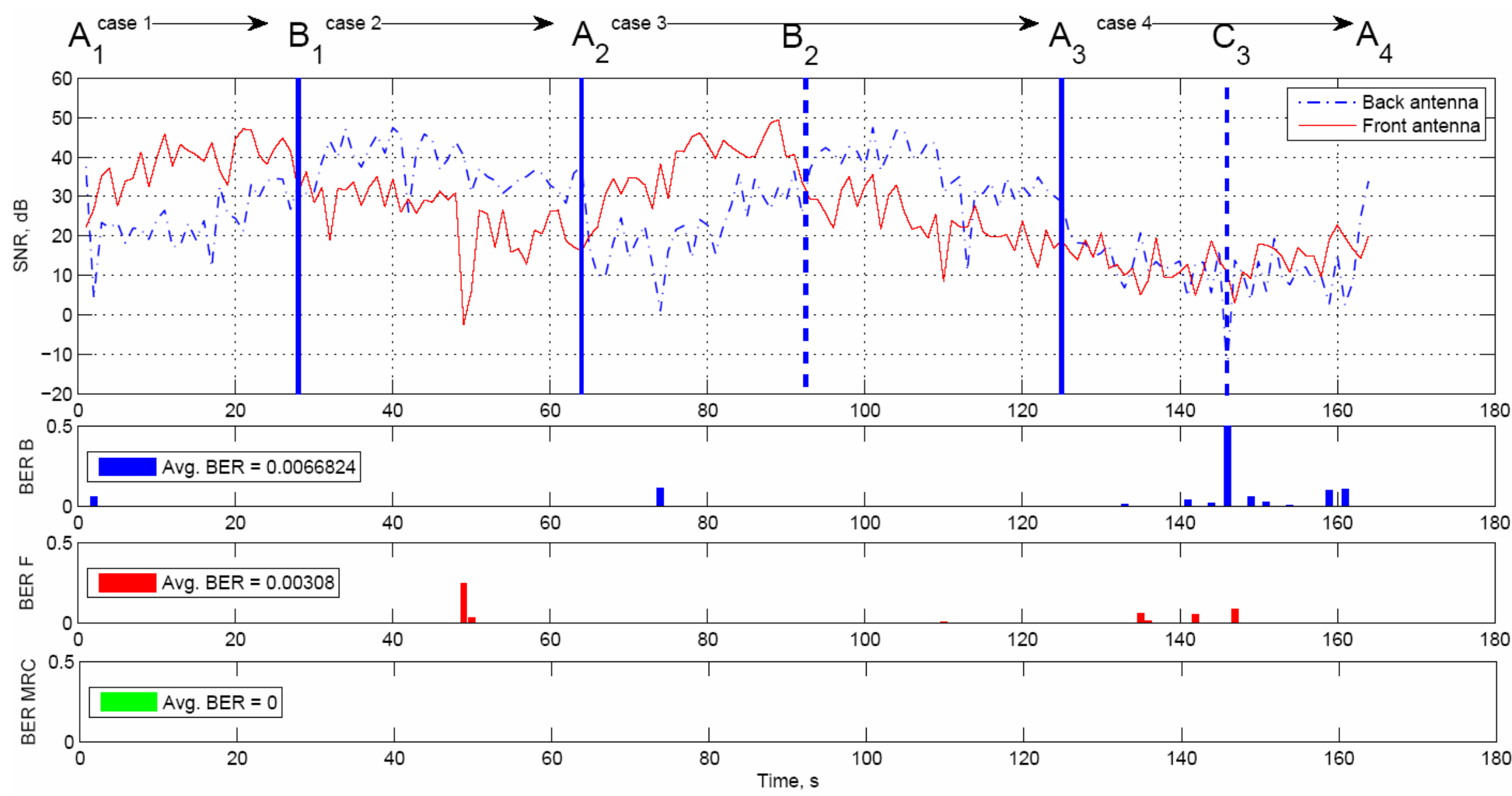


\section{Textile antenna performance}

\section{- Fireman-down scenario}
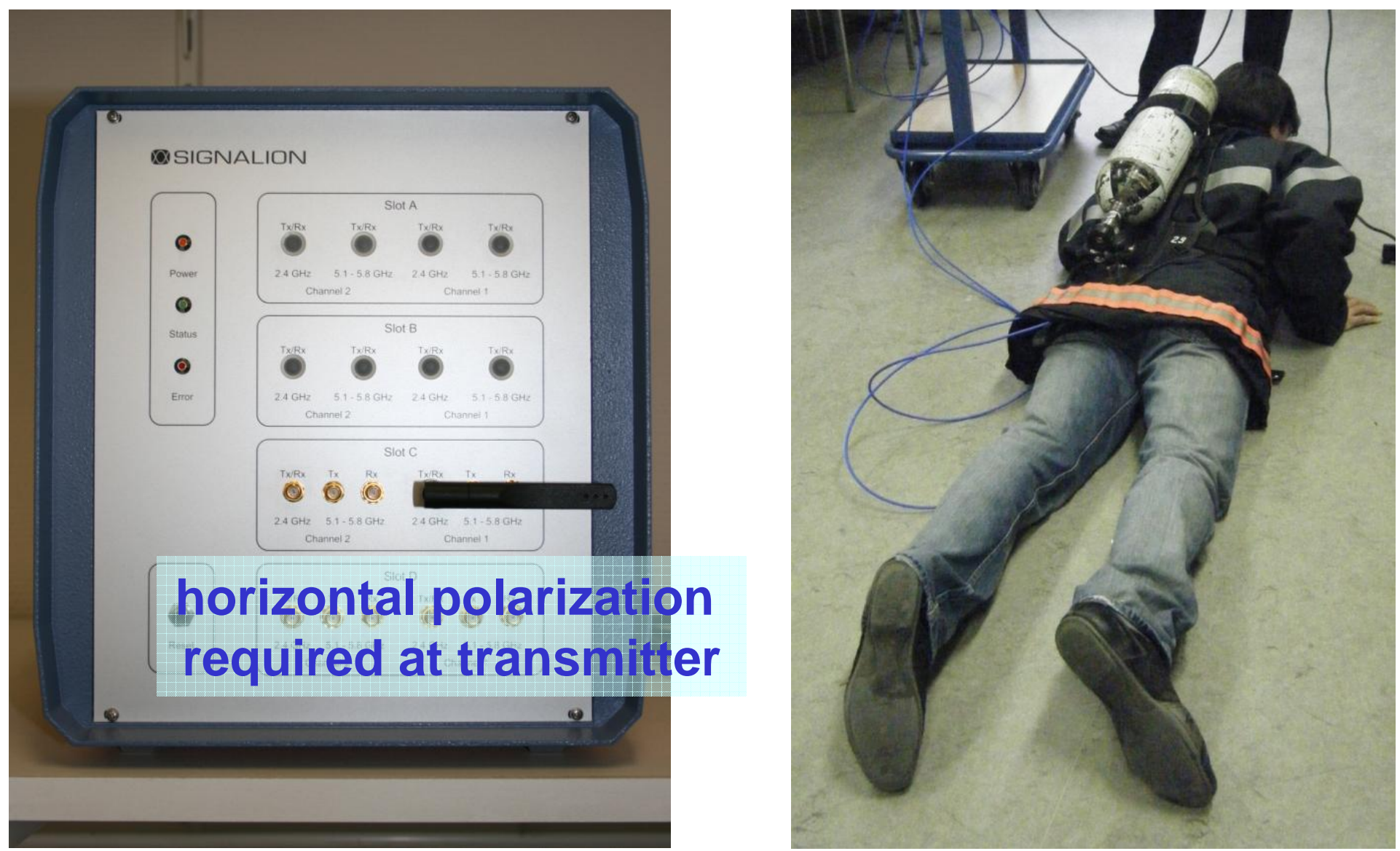


\section{Textile antenna performance}

\section{- Real-life scenario : conclusions}

- Dual textile-antenna system

- Reliable communication by

1. no shadowing by body by placing one antenna at the front and one at the back

2. limited effect of movement and equipment on antenna performance by choosing suitable locations and by careful textile antenna design

3. mitigating fading by exploiting second-order receiver diversity on body

4. careful choice of antenna polarizations

a) circular polarization for on-body antennas avoids need for alignment

b) horizontal polarization at transmitter detects fireman down 


\section{Acknowledgement}

\section{- This research was performed in collaboration with}

- Department of Textiles, UGent

- DIGCOM Group (prof. M. Moeneclaey), Department of Telecommunications and Information Processing, UGent

- financial support of EU
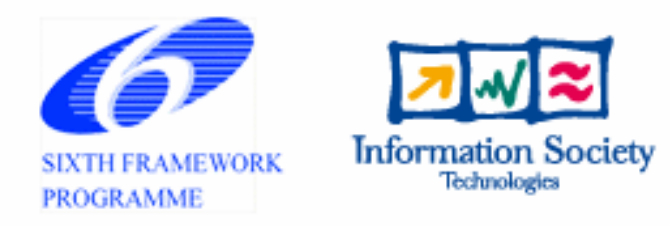


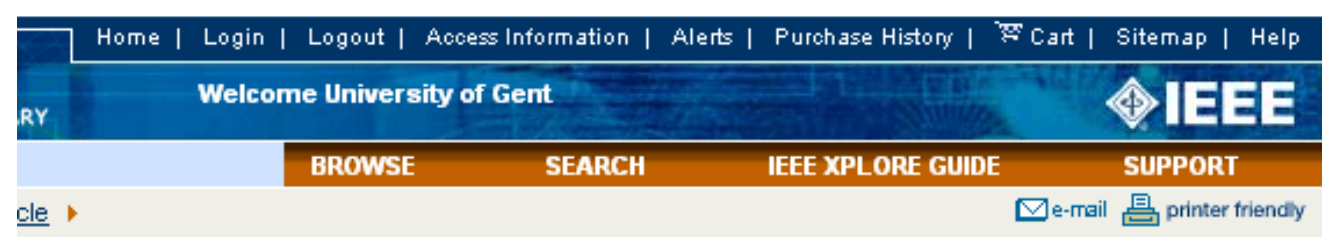

Indoor off-body communication based on a textile multi-antenna system integrated in clothing for rescue workers

Rogier, H. Hertleer, C. Vallozzi, L. Van Torre, P. Declercq, F. Moeneclaey, M.

Inf. Technol. Dept., Ghent Univ., Ghent, Belgium

This paper appears in: Antennas and Propagation for Body-Centric Wireless Communications, 2009 2nd IET Seminar on

Publication Date: 20-20 April 2009

On page(s): 1 - 36

Location: London

ISSN: $0537-9989$

ISBN: $978-1-84919-129-6$

Current Version Published: 2009-06-26

\section{Abstract}

The paper presents a collection of slides that deals with indoor off-body communication based on a textile multiantenna system integrated in clothing for rescue workers. It discusses the design and performance of circularpolarized and dual-polarized textile antennas. 